Contemporary Mathematics

\title{
Statistical ensembles and density of states
}

\author{
Vadim Kostrykin and Robert Schrader
}

Dedicated to Yakov Sinai and David Ruelle on the occasion of their 65th birthday

\begin{abstract}
We propose a definition of microcanonical and canonical statistical ensembles based on the concept of density of states. This definition applies both to the classical and the quantum case. For the microcanonical case this allows for a definition of a temperature and its fluctuation, which might be useful in the theory of mesoscopic systems. In the quantum case the concept of density of states applies to one-particle Schrödinger operators, in particular to operators with a periodic potential or to random Anderson type models. In the case of periodic potentials we show that for the resulting $n$-particle system the density of states is $[(n-1) / 2]$ times differentiable, such that like for classical microcanonical ensembles a (positive) temperature may be defined whenever $n \geq 5$. We expect that a similar result should also hold for Anderson type models. We also provide the first terms in asymptotic expansions of thermodynamic quantities at large energies for the microcanonical ensemble and at large temperatures for the canonical ensemble. A comparison shows that then both formulations asymptotically give the same results.
\end{abstract}

\section{Introduction}

The classical concepts of canonical and grand canonical statistical ensembles are well known to have important quantum analogs (see, e.g., standard textbooks like [50, 35, 64]). To the best of our knowledge the notion of a microcanonical ensemble in quantum theory has not received the attention we think is deserves. This is unfortunate in view of recent developments in the classical and quantum theory of small ensembles. So far the main motivation for a microcanonical analysis of small, classical systems came from gravitational physics with its long range forces (see, e.g., [2, 3, 51, 75, 32, 52, 63, and the references quoted there) and from nuclear physics with the associated fragmentation processes (see, e.g., [28, 29] and the references therein). The main aim was to ask for the most probable distribution and to view this as an equilibrium phenomenon. By energy conservation for closed systems it is natural to consider equilibria in the microcanonical description, where the temperature $T$ and its fluctuation are derived quantities. For the operational definition of a temperature for small systems one cannot use a heat bath but rather a small thermometer, see [75, p.351].

1991 Mathematics Subject Classification. (2000 Revision) Primary 47A40, 82B10; Secondary 82B05, 82D20.

Key words and phrases. Statistical mechanics, ensembles, scattering theory.

The second author is supported in part by DFG SFB 288 "Differentialgeometrie und Quantenphysik".

(c) 0000 (copyright holder) 
Usually one introduces the notion of statistical ensembles to describe the systems with large number of particles. Our approach will in some sense go in a different direction. We deal with the question how small the system can be to allow a thermodynamic description.

We recall that in the familiar classical microcanonical description $1 / T$ may be written as a mean over the energy shell of a certain function in the (finite dimensional) phase space (see relation (2.5) below). Considering the square of this function one may in addition consider fluctuations of the inverse temperature. In case the ergodic hypothesis holds such averages may then be written as time averages using the dynamics of the classical Hamilton function (see, e.g., [67, 68, 5, 60]). An important feature of a microcanonical ensemble is that the temperature may decrease with energy giving rise to a negative heat capacity. The first references which envisage such a situation seem to be [50] and [30]. The example considered in [50] is that of a star, which has used up its nuclear fuel and then heats up under gravitational contraction. A first model discussion for a supernovae providing a negative specific heat was given in [75]. For recent experiments on small systems, see, e.g., [28, 69, 70, 6] and references given there. For more recent theoretical discussions of small systems, see, e.g., [37, 54]. We mention also the recent work [54] where a system of classical particles with power law potentials was considered. The results of this work indicate that already for particle numbers larger than 10 there might be a relaxation to the microcanonical equilibrium starting from an arbitrary initial state.

It is the purpose of this article to provide a useful quantum theoretical approach to the theory of canonical and microcanonical ensembles which may be applied to small (i.e., mesoscopic) systems. This will include a discussion of fluctuations, the heat capacity and their interrelation. We also compare the microcanonical and the canonical ensemble. Our approach will be based on the theory of (integrated) density of states, which in the quantum case is a well known notion in solid state physics (see, e.g., [4, 55]). This theory has been investigated intensively in the last years by mathematical physicists in the context of one-particle Schrödinger operators, in particular, operators with a periodic potential and random Schrödinger operators like Anderson type models. For recent references consult, e.g., [46, 13, 48, 33] and for references before 1992 [27, 41, 10, 61].

In our approach the microcanonical description is given by defining the entropy to be $k$ (the Boltzmann constant) times the logarithm of the density of states, so that the entropy comes to be a function of the energy. As usual the inverse temperature $1 / T$ is then the derivative of the entropy, i.e., $k$ times the logarithmic derivative of the density of states. The canonical description on the other hand is by definition given in terms of a partition function, now defined to be the Stieltjes-Laplace transform of the integrated density of states w.r.t. the variable $\beta=1 / k T$ canonically conjugate to the energy.

In particular we will address the familiar question under which conditions these two descriptions give approximately the same answer. In fact, we will see that the well known methods of comparison in the standard formulation easily carry over to this new formulation. In addition we will show, both in the classical and quantum cases, that for high energies or correspondingly high temperatures the microcanonical and the canonical description give the same results.

So for our approach to work in the microcanonical case, differentiability of the integrated density of states up to third order is necessary. Now our main observation is that smoothness of the integrated density of states for the resulting $n$-particle theory increases with $n$. This will in particular allow us to consider microcanonical ensembles for particles moving in a periodic external potential provided $n \geq 5$. The reason is that for one-particle Schrödinger operators with a periodic potential the density of states (which is the derivative 
of the integrated density of states) is well known to exhibit so called van Hove singularities in space dimensions greater or equal to two (see, e.g., [4, 55]). In one dimension the integrated density of states itself has square root singularities. These are smoothed out when going to higher particle number. We expect a similar property to hold for particles moving in an external random potential. We will argue why increased smoothness with $n$ is related to the well known increasing smoothness of the integrated density of states with the dimension of the space. For Anderson-type random Schrödinger operators the existence of the density of states (i.e., the absolute continuity of the integrated density of states) is a complicated, still not completely solved problem. Very little known is known about regularity properties of the density of states [12, 13, 48, 33.

In addition we will invoke notions from scattering theory and, in particular, the scattering phase shift (or total scattering phase) at a fixed energy $E$. It is an old observation of Beth and Uhlenbeck [7] that the second virial coefficient in statistical mechanics is related to the phase shift (see also [35] and relations (3.8) and (3.24) below). We will use the concept of the phase shift density introduced in [45, 46] to give the asymptotic behavior at large energies (or temperatures) of the main thermodynamic quantities. In particular, in [45, 46] we established that up to a factor of $\pi$ this phase shift density is the difference of the integrated density of states with potential and the free theory. This relation is analogous to the change of the number of particle states found by Friedel [20, 21] in the case of a single impurity. The phase shift density will be used to establish relations on the shift of the temperature induced by the potential in the microcanonical context and on the shift of the mean energy in the canonical context. Furthermore, we establish a relation on the shift of the mean energy density for a system of noninteracting electrons moving in a periodic or a random potential. This relation is analogous to a theorem of Fumi [23] which relates the shift of the ground state energy due to a single impurity to the scattering phase.

We will not discuss situations where in addition to energy other quantities, like, e.g., angular momentum, are conserved. Also we will not cover the situation where in addition to an external potential there is interaction between the particles. This is of course an important issue worth pursuing. We note that non-relativistic $n$-particle scattering theory with all possible fragmentation and bound state channels has been extensively analyzed (see, e.g., [26, 15, 36] and the references quoted there). We expect that some of our results may be extended to this situation, although at present it is unclear to us, which rôle such multichannel spectral properties play in the theory of integrated density of states.

In addition, it would be interesting to see whether the present approach to the theory of quantum mechanical, microcanonical ensembles could serve as a laboratory for a fresh look at ergodic theory in quantum mechanics (see, e.g., [58, 62, 19]).

The article is organized as follows. In Section 2 we will review the classical theory of microcanonical and canonical ensembles in a form which serves as a motivation and comparison for our quantum mechanical approach to be given in Section 3. For the classical models we consider, we will introduce a classical notion of (integrated) density of states and formulate the resulting theory of statistical ensembles. We will see that in this classical setup differentiability indeed increases with the particle number. In addition, we will provide a new example for a system with negative heat capacity. Also we will provide an example where the microcanonical and the canonical descriptions asymptotically for large $E$ and $T$ give the same (mean) energy - temperature relation. Recall that in the usual canonical description the heat capacity is always positive.

So the discussion in Section 2 will be rather extensive, the reason being that the quantum theory is then simply obtained by replacing the classical (integrated) density of states by the corresponding quantum version. 
Section 3 will contain the corresponding quantum mechanical formulation of both the microcanonical and the canonical ensemble using the quantum notion of the integrated density of states. For large energies we again will argue on the basis of a yet unproven conjecture on the high energy behavior of the phase shift [71] that the (mean) energy temperature relations are asymptotically equal for both ensembles. As already mentioned we view this result as an indication of the reasonableness of our thermodynamic approach to small systems. Section 3 will also include a brief comparison between the classical and the quantum theory for small $\hbar$. Finally in Section 3 we also briefly discuss the grand canonical ensemble in terms of the integrated density of states.

In the appendix we will show in the quantum context for periodic potentials that singularities of the $n$-particle density of states are smoothed out with increasing number of particles $n$. In addition we will show in the classical case how the randomness in stochastic potentials smoothes out the integrated density of states.

We avoid to formulate our results as theorems and propositions. However making any statement we provide a discussion of what is rigorously proven and what is only conjectured motivated by the physical intuition.

Acknowledgments. We are indebted to R.M. Dudley, P.D. Hislop, M. Karowski, H. Narnhofer, M. Schmidt, K.-D. Schotte, H. Spohn, and W. Thirring for valuable comments. We thank D.H.E. Gross and O. Fliegans for providing references.

\section{The classical theory}

In this section and for the purpose of comparison and as part of our motivation we will briefly review the well known concepts of microcanonical and canonical ensembles in the classical case. Some of the material, however, seems to be new. In particular we will provide new examples with negative heat capacity. Previous examples in the context of gravitation theory are given, e.g., [52, 75] and the references quoted there.

In classical theory the starting point is a finite dimensional phase space $\mathcal{P}$ which is a symplectic manifold. We will assume, in addition, that $\mathcal{P}$ also is a Kähler manifold, i.e., it is also a Riemannian manifold and the symplectic and Riemanian structures are compatible. This implies, in particular, that the Liouville volume form and Riemannian volume form agree. We denote this volume form by $d \mathrm{vol}_{\mathcal{P}}$. Also a Hamilton function $H_{\mathrm{cl}}$ on $\mathcal{P}$ is supposed to be smooth and bounded below in case $\mathcal{P}$ is not compact.

First we assume $\mathcal{P}_{\leq E}=\left\{p \in \mathcal{P} \mid H_{\mathrm{cl}}(p) \leq E\right\}$ is compact for all $E$. Note that Hamilton functions of the form $H_{\mathrm{cl}}(p, x)=p^{2} / 2 m+V(x)$ with bounded $V$ do not satisfy this assumption. We will turn to this later.

The function

$$
N\left(E ; H_{\mathrm{cl}}\right)=\int_{\mathcal{P}_{\leq E}} d \operatorname{vol}_{\mathcal{P}}=\int_{\mathcal{P}} \Theta\left(E-H_{\mathrm{cl}}\right) d \operatorname{vol}_{\mathcal{P}},
$$

where $\Theta$ is the Heaviside step function, is increasing w.r.t. the energy $E$. Its derivative is given as

$$
0 \leq W\left(E ; H_{\mathrm{cl}}\right)=\frac{d}{d E} N\left(E ; H_{\mathrm{cl}}\right)=\int_{\mathcal{P}} \delta\left(E-H_{\mathrm{cl}}\right) d \operatorname{vol}_{\mathcal{P}}
$$

with $\delta$ being the Dirac $\delta$-function. The associated microcanonical ensemble is then given by defining the entropy as a function of the energy $E$ as $S\left(E ; H_{\mathrm{cl}}\right)=k \ln W\left(E ; H_{\mathrm{cl}}\right)$ and the temperature $T(E)=T\left(E ; H_{\mathrm{cl}}\right)$, again considered as a function of the energy $E$, is then 
defined by

$$
\frac{1}{T(E)}=\frac{d S\left(E ; H_{\mathrm{cl}}\right)}{d E}=k \frac{d}{d E} \ln \frac{d N\left(E ; H_{\mathrm{cl}}\right)}{d E}=k \frac{1}{W\left(E ; H_{\mathrm{cl}}\right)} \frac{d W\left(E ; H_{\mathrm{cl}}\right)}{d E}
$$

with $k$ being the Boltzmann constant. So provided the r.h.s. of (2.2) is meaningful the temperature is defined but possibly negative. In standard situations $W\left(E ; H_{\mathrm{cl}}\right)$ increases with $E$ such that $T$ is nonnegative. However, there are also situations, where the temperature $T$ may become negative (see, e.g., [50]).

The function $W\left(E ; H_{\mathrm{cl}}\right)$ and its two first derivatives may be written in the form

$$
\begin{aligned}
W\left(E ; H_{\mathrm{cl}}\right) & =\int_{\mathcal{P}_{E}} d \mu_{E} \\
\frac{d}{d E} W\left(E ; H_{\mathrm{cl}}\right) & =\int_{\mathcal{P}_{E}} \nabla \cdot \frac{\nabla H_{\mathrm{cl}}}{\left|\nabla H_{\mathrm{cl}}\right|^{2}} d \mu_{E} \\
\frac{d^{2}}{d E^{2}} W\left(E ; H_{\mathrm{cl}}\right) & =\int_{\mathcal{P}_{E}} \nabla \cdot\left(\left(\nabla \cdot \frac{\nabla H_{\mathrm{cl}}}{\left|\nabla H_{\mathrm{cl}}\right|^{2}}\right) \frac{\nabla H_{\mathrm{cl}}}{\left|\nabla H_{\mathrm{cl}}\right|^{2}}\right) d \mu_{E}
\end{aligned}
$$

with the following notation. The manifold $\mathcal{P}_{E}$, the boundary of $\mathcal{P}_{\leq E}$, is the energy surface for the energy $E$, i.e., the set of points $p \in \mathcal{P}$ for which $H_{\mathrm{cl}}(p)=E$. The measure $d \mu_{E}$ on $\mathcal{P}_{E}$ is given as $d \mu_{E}=\left|\nabla H_{\mathrm{cl}}\right|^{-1} d \operatorname{vol}_{\mathcal{P}_{E}}$, where $d \operatorname{vol}_{\mathcal{P}_{E}}$ is the canonical volume form on $\mathcal{P}_{E}$ given by the Riemannian metric. $\nabla$ is the covariant gradient and $\left|\nabla H_{\mathrm{cl}}\right|(p)$ the length of the vector $\nabla H_{\mathrm{cl}}(p)$, which is normal to the surface $\mathcal{P}_{E}$ at $p \in \mathcal{P}_{E}$. So provided $\nabla H_{\mathrm{cl}}(p) \neq 0$, the vector $1 /\left|\nabla H_{\mathrm{cl}}(p)\right| \nabla H_{\mathrm{cl}}(p)$ is the outward unit normal vector to $\mathcal{P}_{E}$ at $p \in \mathcal{P}_{E}$, i.e., this vector points into the complement $\mathcal{P}_{>E}$ of $\mathcal{P}_{<E}$. Near $\mathcal{P}_{E}$ one has the familiar relation $d \operatorname{vol}_{\mathcal{P}}=d \mu_{E} d E$, whence the first relation in (2.3). Finally $\nabla \cdot$ is the covariant divergence. The second and third relation in (2.3) are obtained from the first by (repeated) use of the theorem of Gauss (see (2.11) below). Higher derivatives may be calculated similarly. In standard situations $\mathcal{P}_{E}$ is a compact set, generically of codimension 1 . For $W_{\mathrm{cl}}\left(E ; H_{\mathrm{cl}}\right)$ and its derivatives to be well defined one has to assume that $\nabla H_{\mathrm{cl}}(p) \neq 0$ for $p \in \mathcal{P}_{E}$ (or at least that suitable inverse powers of $\left|\nabla H_{\mathrm{cl}}\right|(p)$ are integrable over $\left.\mathcal{P}_{E}\right)$. Then in particular $\mathcal{P}_{E}$ is a smooth submanifold of $\mathcal{P}$ of codimension 1 and $W\left(E ; H_{\mathrm{cl}}\right) \neq 0$ provided the energy $E$ is such that $\mathcal{P}_{E} \neq \varnothing$. The first relation in (2.3) dates back to Khinchin [42].

The temperature $T\left(E ; H_{\mathrm{cl}}\right)$ defined by 2.2$)$ is an increasing function at $E$ if

$$
W\left(E ; H_{\mathrm{cl}}\right) \frac{d^{2}}{d E^{2}} W\left(E ; H_{\mathrm{cl}}\right) \leq\left(\frac{d}{d E} W\left(E ; H_{\mathrm{cl}}\right)\right)^{2} \text {. }
$$

We may rephrase this as follows (see also [67, 68]). Let

$$
\langle f\rangle_{E}=\frac{\int_{\mathcal{P}_{E}} f d \mu_{E}}{\int_{\mathcal{P}_{E}} d \mu_{E}}=\frac{\int \delta\left(E-H_{\mathrm{cl}}\right) f d \operatorname{vol}_{\mathcal{P}}}{\int \delta\left(E-H_{\mathrm{cl}}\right) d \operatorname{vol}_{\mathcal{P}}}
$$

denote the average over the energy shell $\mathcal{P}_{E}$ of a real valued function $f$ defined on $\mathcal{P}_{E}$. For given $H_{\mathrm{cl}}$ consider in particular the function $\mathcal{T}$ on $\mathcal{P}$ of the dimension of energy given as

$$
\mathcal{T}^{-1}=\nabla \cdot \frac{\nabla H_{\mathrm{cl}}}{\left|\nabla H_{\mathrm{cl}}\right|^{2}}
$$

such that

$$
\frac{1}{T(E)}=k\left\langle\mathcal{T}^{-1}\right\rangle_{E}
$$

In general $k T(E) \neq\langle\mathcal{T}\rangle_{E}$. Consider for example the case where $0<\mathcal{T}<\infty$ on $\mathcal{P}_{E}$. Then

$$
1=\langle 1\rangle_{E}=\left\langle\mathcal{T}^{1 / 2} \mathcal{T}^{-1 / 2}\right\rangle_{E} \leq\langle\mathcal{T}\rangle_{E}^{1 / 2}\left\langle\mathcal{T}^{-1}\right\rangle_{E}^{1 / 2}
$$


by the Schwarz inequality giving $\langle\mathcal{T}\rangle_{E} \geq k T(E)$ with equality if and only if $\mathcal{T}$ is constant on $\mathcal{P}_{E}$.

We write

$$
\begin{aligned}
& \nabla \cdot\left(\left(\nabla \cdot \frac{\nabla H_{\mathrm{cl}}}{\left|\nabla H_{\mathrm{cl}}\right|^{2}}\right) \frac{\nabla H_{\mathrm{cl}}}{\left|\nabla H_{\mathrm{cl}}\right|^{2}}\right)=\frac{1}{\mathcal{T}^{2}}+\mathcal{G}, \\
& \mathcal{G}=\left(\nabla\left(\nabla \cdot \frac{\nabla H_{\mathrm{cl}}}{\left|\nabla H_{\mathrm{cl}}\right|^{2}}\right)\right) \cdot \frac{\nabla H_{\mathrm{cl}}}{\left|\nabla H_{\mathrm{cl}}\right|^{2}}=\left(\nabla \mathcal{T}^{-1}\right) \cdot \frac{\nabla H_{\mathrm{cl}}}{\left|\nabla H_{\mathrm{cl}}\right|^{2}} .
\end{aligned}
$$

Here - denotes the scalar product of two vectors (or rather vector fields) given by the Riemannian metric, such that in particular $\left|\nabla H_{\mathrm{cl}}\right|^{2}=\nabla H_{\mathrm{cl}} \cdot \nabla H_{\mathrm{cl}}$.

Consider the quantity $\Delta\left(T^{-1}\right)(E)=\Delta\left(T^{-1}\right)\left(E ; H_{\mathrm{cl}}\right)$ given by

$$
\Delta\left(T^{-1}\right)(E)^{2}=k^{2}\left(\left\langle\mathcal{T}^{-2}\right\rangle_{E}-\left\langle\mathcal{T}^{-1}\right\rangle_{E}^{2}\right) \geq 0
$$

may be viewed as the fluctuation of the inverse temperature at energy $E$. Note that the inequality is a consequence of the Schwarz inequality or equivalently of the familiar relation $0 \leq\left\langle\left(f-\langle f\rangle_{E}\right)^{2}\right\rangle_{E}$. In particular $\Delta\left(T^{-1}\right)(E)$ vanishes if and only if $\mathcal{T}$ is constant on $\mathcal{P}_{E}$. The fluctuation and the heat capacity $c_{\mathrm{v}}(E) \equiv c_{\mathrm{v}}\left(E ; H_{\mathrm{cl}}\right)$ defined by

$$
\frac{1}{c_{\mathrm{v}}(E)}=\frac{d}{d E} T(E)=-T(E)^{2} \frac{d}{d E} \frac{1}{T(E)}=-k T(E)^{2} \frac{d}{d E} \ln \frac{d}{d E} W\left(E ; H_{\mathrm{cl}}\right)
$$

are related by

$$
\Delta\left(T^{-1}\right)(E)^{2}+\frac{k}{T(E)^{2} c_{\mathrm{v}}(E)}=-k^{2}\langle\mathcal{G}\rangle_{E} .
$$

Therefore condition (2.4), which guarantees that $c_{\mathrm{v}}(E) \geq 0$, may be recast into the equivalent form

$$
\frac{1}{k^{2}} \Delta\left(T^{-1}\right)(E)^{2} \leq-\langle\mathcal{G}\rangle_{E}
$$

By (2.6) we have $\left|\nabla H_{\mathrm{cl}}\right| \mathcal{G}=\nabla(1 / \mathcal{T}) \cdot\left|\nabla H_{\mathrm{cl}}\right|^{-1} \nabla H_{\mathrm{cl}}$. This has the following geometric interpretation. $\left|\nabla H_{\mathrm{cl}}\right| \mathcal{G}$ is the component of the gradient of $1 / \mathcal{T}$ in the normal direction. So if this gradient always points into $\mathcal{P}_{\leq E}$ (i.e., $\nabla(1 / \mathcal{T}) \cdot \nabla H_{\mathrm{cl}}(s) \leq 0$ for all $s \in \mathcal{P}_{E}$ ) thus making $\mathcal{G}$ negative (or zero) there, then the inequality (2.10) is satisfied for the energy $E$. Since $\nabla(1 / \mathcal{T})=-(1 / \mathcal{T})^{2} \nabla \mathcal{T}$ this is equivalent to the condition that the gradient of $\mathcal{T}$ always points into $\mathcal{P}_{>E}=\mathcal{P} \backslash \mathcal{P}_{\leq E}$. Conversely, if the inequality in (2.10) is reversed and in particular if $\langle\mathcal{G}\rangle_{E}>0$ (meaning that in the mean the gradient of $1 / \mathcal{T}$ points into $\mathcal{P}_{>E}$, then $T\left(E, H_{\mathrm{cl}}\right)$ is a monotone decreasing function in $E$, i.e., the heat capacity is negative.

By means of the Gauss theorem and using (2.6) the relations (2.3) can be written equivalently as follows

$$
\begin{aligned}
W\left(E ; H_{\mathrm{cl}}\right) & =\int_{\mathcal{P}_{\leq E}} \mathcal{T}^{-1} d \operatorname{vol}_{\mathcal{P}}, \\
\frac{d}{d E} W\left(E ; H_{\mathrm{cl}}\right) & =\int_{\mathcal{P}_{\leq E}}\left(\mathcal{T}^{-1}+\mathcal{T} \mathcal{G}\right) \mathcal{T}^{-1} d \operatorname{vol}_{\mathcal{P}}, \\
\frac{d^{2}}{d E^{2}} W\left(E ; H_{\mathrm{cl}}\right) & =\int_{\mathcal{P}_{\leq E}}\left(\mathcal{T}^{-2}+2 \mathcal{G}+\mathcal{T} \nabla \mathcal{G} \cdot \frac{\nabla H_{\mathrm{cl}}}{\left|\nabla H_{\mathrm{cl}}\right|^{2}}\right) \mathcal{T}^{-1} d \operatorname{vol}_{\mathcal{P}}
\end{aligned}
$$

Moreover, for any differentiable function $f$ on $\mathcal{P}_{\leq E}$ the average over the energy shell can be written as

$$
\langle f\rangle_{E}=\frac{\int_{\mathcal{P}_{\leq E}}\left(\nabla f \cdot \frac{\nabla H_{\mathrm{c} \mid}}{\left.\nabla \nabla H_{\mathrm{c}}\right|^{2}}+f \mathcal{T}^{-1}\right) d \operatorname{vol}_{\mathcal{P}}}{\int_{\mathcal{P}_{\leq E}} \mathcal{T}^{-1} d \operatorname{vol}_{\mathcal{P}}} .
$$


Assume now that $\mathcal{T}>0$ on $\mathcal{P}_{\leq E}$. Then we may introduce the probability measure on $\mathcal{P}_{\leq E}$ by

$$
d v_{E}=\frac{1}{W\left(E ; H_{\mathrm{cl}}\right)} \Theta\left(E-H_{\mathrm{cl}}\right) \mathcal{T}^{-1} d \operatorname{vol}_{\mathcal{P}}
$$

In analogy to $\langle\cdot\rangle_{E}$ let $\langle\cdot\rangle_{E}^{\prime}$ denote the resulting mean. Then relations $(2.5)$ can be rewritten as

$$
\frac{1}{T\left(E ; H_{\mathrm{cl}}\right)}=k\left\langle\mathcal{T}^{-1}+\mathcal{T} \mathcal{G}\right\rangle_{E}^{\prime}
$$

However, the resulting inverse temperature fluctuation given by

$$
\Delta^{\prime}\left(T^{-1}\right)(E)=\left(k^{2}\left\langle\left(\mathcal{T}^{-1}+\mathcal{T} \mathcal{G}\right)^{2}\right\rangle_{E}^{\prime}-T(E)^{-2}\right)^{1 / 2}
$$

is in general different from $\Delta\left(T^{-1}\right)(E)$. In fact, by 2.12 and the Gauss theorem

$$
0 \leq\left\langle\mathcal{T}^{-2}\right\rangle_{E}=\left\langle\left(\mathcal{T}^{-2}+2 \mathcal{G}\right)\right\rangle_{E}^{\prime}=\left\langle\left(\mathcal{T}^{-1}+\mathcal{T G}\right)^{2}\right\rangle_{E}^{\prime}-\left\langle(\mathcal{T G})^{2}\right\rangle_{E}^{\prime}
$$

This implies $\Delta\left(T^{-1}\right)(E) \leq \Delta^{\prime}\left(T^{-1}\right)(E)$ which is an equality if and only if $\mathcal{T G}$ vanishes identically on $\mathcal{P}_{\leq E}$. Relation (2.9) is now replaced by

$$
\Delta^{\prime}\left(T^{-1}\right)(E)^{2}+\frac{k}{T(E)^{2} c_{\mathrm{v}}(E)}=-\left\langle\mathcal{T} \nabla \mathcal{G} \cdot \frac{\nabla H_{\mathrm{cl}}}{\left|\nabla H_{\mathrm{cl}}\right|^{2}}\right\rangle_{E}^{\prime},
$$

so alternatively to 2.10 the condition 2.4 for the heat capacity to be positive can now be written as

$$
\frac{1}{k^{2}} \Delta^{\prime}\left(T^{-1}\right)(E)^{2} \leq-\left\langle\mathcal{T} \nabla \mathcal{G} \cdot \frac{\nabla H_{\mathrm{cl}}}{\left|\nabla H_{\mathrm{cl}}\right|^{2}}\right\rangle_{E}^{\prime} .
$$

The alternative definition (2.14) of the inverse temperature fluctuation has the disadvantage that besides the assumed positivity of $\mathcal{T}$ it involves the values of $H_{\mathrm{cl}}$ on all of $\mathcal{P}_{\leq E}$. In contrast and due to the appearance of derivatives of $H_{\mathrm{cl}}$, the definition $(2.7)$ only involves the values of $H_{\mathrm{cl}}$ near $\mathcal{P}_{E}$.

The Boltzmann-Gibbs partition function in the associated canonical ensemble may also be given in terms of the functions $N\left(E ; H_{\mathrm{cl}}\right)$ and $W\left(E ; H_{\mathrm{cl}}\right)$ as

$$
Z\left(\beta ; H_{\mathrm{cl}}\right)=\int e^{-\beta H_{\mathrm{cl}}} d \operatorname{vol}_{\mathcal{P}}=\int e^{-\beta E} d N\left(E ; H_{\mathrm{cl}}\right)=\int e^{-\beta E} W\left(E ; H_{\mathrm{cl}}\right) d E, \quad \beta=1 / k T .
$$

The (mean) energy-temperature relation now takes the form

$$
\bar{E}\left(\beta ; H_{\mathrm{cl}}\right)=-\frac{d}{d \beta} Z\left(\beta ; H_{\mathrm{cl}}\right)=\langle E\rangle_{\beta} \quad \text { with } \quad\langle g\rangle_{\beta}=\frac{\int g(E) e^{-\beta E} W\left(E ; H_{\mathrm{cl}}\right) d E}{Z\left(\beta ; H_{\mathrm{cl}}\right)}
$$

being the mean of any function $g$ of the energy for given $\beta$. The mean energy now is a monotone increasing function of temperature. Since $\left\langle E^{2}\right\rangle_{\beta} \geq\langle E\rangle_{\beta}^{2}$ by the Schwarz inequality, $\Delta \bar{E}\left(\beta ; H_{\mathrm{cl}}\right)=\left(\left\langle E^{2}\right\rangle_{\beta}-\langle E\rangle_{\beta}^{2}\right)^{1 / 2}$ gives the energy fluctuation. Stated differently, the heat capacity defined in this canonical context as

$$
c_{V}\left(\beta ; H_{\mathrm{cl}}\right)=\frac{\partial}{\partial T} \bar{E}\left(\beta ; H_{\mathrm{cl}}\right)=-\frac{\beta^{2}}{k} \frac{\partial}{\partial \beta} \bar{E}\left(\beta ; H_{\mathrm{cl}}\right)
$$

is always nonnegative. 
The following discussion (see also [68]) relates the microcanonical and canonical descriptions. It is an adaptation of well known arguments employed in this context (see, e.g., [35]) and is recalled for later purpose. Write

$$
Z\left(\beta ; H_{\mathrm{cl}}\right)=\int e^{-\beta E+\ln \frac{1}{\beta} W\left(E ; H_{\mathrm{cl}}\right)} d E .
$$

The integrand takes an extremal value at $E_{\max }=E_{\max }(T)$ given implicitly by

$$
\frac{1}{T}=\left.k \frac{d}{d E} \ln W\left(E ; H_{\mathrm{cl}}\right)\right|_{E=E_{\max }},
$$

which agrees with (2.2). If a solution $E_{\max }$ to the relation 2.18) exists it is unique. If the inequality (2.4) holds for all $E$ and is a strict inequality at $E=E_{\max }$ then the extremal value of the integrand is a maximal one. Indeed, for $E$ near $E_{\max }$ we obtain

$$
-\beta E+\ln \left(\frac{1}{\beta} W\left(E ; H_{\mathrm{cl}}\right)\right)=-\frac{1}{2} \alpha\left(E-E_{\max }\right)^{2}+O\left(\left(E-E_{\max }\right)^{3}\right)
$$

with $\alpha=\alpha\left(\beta ; H_{\mathrm{cl}}^{(n)}\right)$ given by $\alpha=-d^{2} \ln W\left(E=E_{\max } ; H_{\mathrm{cl}}\right) / d E^{2}$, i.e.,

$$
\alpha=\left.\frac{\left(\frac{d}{d E} W\left(E ; H_{\mathrm{cl}}\right)\right)^{2}-\frac{d^{2}}{d E^{2}} W\left(E ; H_{\mathrm{cl}}\right) \cdot W\left(E ; H_{\mathrm{cl}}\right)}{W\left(E ; H_{\mathrm{cl}}\right)^{2}}\right|_{E=E_{\max }} .
$$

which is nonnegative if (2.4) holds and is strictly positive if (2.4) is a strict inequality at $E=E_{\max }$. In fact, in the microcanonical notation $\alpha$ is related to the heat capacity via $\alpha=1 /\left(k c_{\mathrm{v}}\left(E_{\max }\right) T\left(E_{\max }\right)^{2}\right)$ by $(2.18)$. So when $\alpha>0$ is large, to a good approximation the integrand in $(2.17)$ is given by a Gaussian distribution with variance $\alpha^{-1}$. The energy fluctuation is then $\Delta \bar{E}\left(\beta ; H_{\mathrm{cl}}\right) \sim \alpha^{-1 / 2}$. Finally if in the canonical ensemble the heat capacity is defined as $c_{\mathrm{v}}(T)=d \bar{E}\left(\beta ; H_{\mathrm{cl}}\right) / d T$ this quantity then agrees with the microcanonical heat capacity at the energy $E_{\max }$, i.e., $k T^{2} c_{\mathrm{v}}(T)=\Delta \bar{E}\left(\beta ; H_{\mathrm{cl}}\right)^{2} \sim k T^{2} c_{\mathrm{v}}\left(E_{\max }\right)$.

¿From now on we will consider classical Hamilton functions with non-compact $\mathcal{P}_{\leq E}$ such that the above discussion does nor apply. The following discussion serves as a preparation to what we will do in the quantum case in the next section. More concretely, we consider a particle of mass $m>0$ moving in $\mathbb{R}^{d}$ under the influence of a periodic bounded potential $V(x)$, i.e., $V(x)=V(x+j)$ for all $x \in \mathbb{R}^{d}$ and all $j \in \mathbb{Z}^{d}$. Thus the classical Hamilton function is given as $H_{\mathrm{cl}}(x, p)=p^{2} / 2 m+V(x)$ and the phase space $\mathcal{P}$ is $\mathbb{R}^{2 d}$ with the canonical symplectic structure. We will consider the resulting $n$-particle theory with phase space $\mathbb{R}^{2 n d}$ and classical Hamilton function $H_{\mathrm{cl}}^{(n)}(x, p)=p^{2} / 2 m+V^{(n)}(x)=H_{0 \mathrm{cl}}^{(n)}(p)+V^{(n)}(x)$. Here $p=\left(p_{1}, p_{2}, \ldots, p_{n}\right), x=\left(x_{1}, x_{2}, \ldots, x_{n}\right), p_{i}, x_{i} \in \mathbb{R}^{d}$ and $V^{(n)}(x)=\sum_{i} V\left(x_{i}\right)$. For the purpose of this article it will suffice to assume the potential to be bounded.

We will also consider random, classical Hamiltonians of the so called Anderson type. More precisely, we assume the one particle random potential $V=V_{\omega}$ to be of the form

$$
V_{\omega}(x)=\sum_{j \in \mathbb{Z}^{d}} q_{j}(\omega) V_{0}(x-j) .
$$

Here $\omega=\left\{\omega_{j}\right\}_{j \in \mathbb{Z}^{d}}$ is an element of the probability space $\Omega=\mathbb{R}^{\mathbb{Z}^{d}}$ with a probability measure Prob and $q_{j}(\omega)=q\left(\omega_{j}\right)$ for some real valued bounded measurable function $q$. Therefore $\omega_{j}$ are independent random variables with the same distribution. In other words there is a probability measure $d \mu$ on $\mathbb{R}$ such that $\operatorname{Prob}\left(q_{j} \in I\right)=\int_{I} d \mu(q)$ for any interval $I \subset \mathbb{R}$ and any $j \in \mathbb{Z}^{d}$. Also we assume that $V_{0}$ is a bounded function with support in the unit cube in $\mathbb{R}^{d}$, such that $V_{0}(x-j)$ and $V_{0}(x-k)$ have non-overlapping support when $j \neq k$. This results 
in a Hamiltonian $H_{\mathrm{cl}}(p, x)=H_{\mathrm{cl}, \omega}(p, x)=p^{2} / 2 m+V_{\omega}^{(n)}(x)$, again with the obvious notation $V_{\omega}^{(n)}(x)=\sum_{i} V_{\omega}\left(x_{i}\right)$.

In the considered cases the function $N\left(E ; H_{\mathrm{cl}}^{(n)}\right)$ defined by 2.1. is infinite but the integrated density of states, given as

$$
\mathfrak{n}\left(E ; H_{\mathrm{cl}}^{(n)}\right)=\lim _{\Lambda \rightarrow \infty} \frac{1}{|\Lambda|^{n}} \int_{\Lambda^{n} \otimes \mathbb{R}^{n d}} \Theta\left(E-H_{\mathrm{cl}}^{(n)}(p, x)\right) d^{n d} x d^{n d} p
$$

exists where $\Lambda \subset \mathbb{R}^{d}$ and $\Lambda^{n}=\Lambda \times \ldots \times \Lambda \subset \mathbb{R}^{n d}$. If $V$ is periodic we obviously have

$$
\begin{aligned}
\mathfrak{n}\left(E ; H_{\mathrm{cl}}^{(n)}\right) & =\int_{\Lambda_{0}^{n} \otimes \mathbb{R}^{n d}} \Theta\left(E-H_{\mathrm{cl}}^{(n)}(p, x)\right) d^{n d} x d^{n d} p \\
& =\frac{1}{n d} \operatorname{Vol}\left(\mathbb{S}^{n d-1}\right)(2 m)^{n d / 2} \int_{\Lambda_{0}^{n}} \Theta\left(E-V^{(n)}(x)\right)\left(E-V^{(n)}(x)\right)^{n d / 2} d^{n d} x .
\end{aligned}
$$

where $\Lambda_{0}$ is the unit cube in $\mathbb{R}^{d}, \mathbb{S}^{n d-1}$ is the unit sphere in $\mathbb{R}^{n d}$ and $\operatorname{Vol}\left(\mathbb{S}^{n d-1}\right)$ its area. In the case of free motion, i.e., $V=0$, and using the fact $\operatorname{Vol}\left(\mathbb{S}^{n d-1}\right)=2 \pi^{n d / 2} / \Gamma(n d / 2)$ we obtain

$$
\mathfrak{n}\left(E ; H_{0 \mathrm{cl}}^{(n)}\right)=\frac{1}{n d} \operatorname{Vol}\left(\mathbb{S}^{n d-1}\right)(2 m E)^{n d / 2}=\frac{(2 \pi m E)^{n d / 2}}{\Gamma(n d / 2+1)}, \quad E \geq 0 .
$$

Note that from 2.21 it follows that the order of differentiability of $\mathfrak{n}\left(E ; H_{\mathrm{cl}}^{(n)}\right)$ w.r.t. $E$ increases with $n d$.

In the random case $V=V_{\omega}$ we obtain in place of (2.21)

$$
\begin{aligned}
\mathfrak{n}\left(E ; H_{\mathrm{cl}}^{(n)}\right)= & \frac{1}{n d} \operatorname{Vol}\left(\mathbb{S}^{n d-1}\right)(2 m)^{n d / 2} \\
& \cdot \int_{\mathbb{R}} d \mu(q) \int_{\Lambda_{0}^{n}} \Theta\left(E-q \sum_{i} V_{0}\left(x_{i}\right)\right)\left(E-q \sum_{i} V_{0}\left(x_{i}\right)\right)^{n d / 2} d^{n d} x .
\end{aligned}
$$

For fixed number of particles $n$ the smoothness of $\mathfrak{n}\left(E ; H_{\mathrm{cl}}^{(n)}\right)$ depends on the probability distribution $d \mu(q)$ of random variables $q_{j}(\omega)$. Assume that the measure $d \mu(q)$ is absolutely continuous with smooth density supported in an interval $\left[\varepsilon, \varepsilon^{-1}\right]$ for some $0<\varepsilon<1$. Then we may rewrite 2.23 as

$$
\begin{aligned}
\mathfrak{n}\left(E ; H_{\mathrm{cl}}^{(n)}\right)= & \frac{1}{n d} \operatorname{Vol}\left(\mathbb{S}^{n d-1}\right)(2 m)^{n d / 2} \\
& \cdot \int q^{n d / 2} f(q) d q \int_{\Lambda_{0}} \Theta\left(\frac{E}{q}-V_{0}(x)\right)\left(\frac{E}{q}-V_{0}(x)\right)^{n d / 2} d^{n d} x \\
= & E^{n d / 2+1} \frac{1}{n d} \operatorname{Vol}\left(\mathbb{S}^{n d-1}\right)(2 m)^{n d / 2} \\
& \cdot \int q^{n d / 2} f(E q) d q \int_{\Lambda_{0}} \Theta\left(\frac{1}{q}-V_{0}(x)\right)\left(\frac{1}{q}-V_{0}(x)\right)^{n d / 2} d^{n d} x .
\end{aligned}
$$

In particular, if $f \in C^{\infty}$ then the degree of differentiability of $\mathfrak{n}\left(E ; H_{\mathrm{cl}}^{(n)}\right)$ is completely determined by the factor $E^{n d / 2+1}$.

The main idea of the present work is to replace $N\left(E ; H_{\mathrm{cl}}^{(n)}\right)$ in the relations 2.1 (2.2) by the integrated density of states $\mathfrak{n}\left(E ; H_{\mathrm{cl}}^{(n)}\right)$. For $n d \geq 3$ this gives the temperature 
$T(E)=T\left(E ; H_{\mathrm{cl}}^{(n)}\right)$ as

$$
\begin{aligned}
\frac{1}{T(E)} & =\frac{d}{d E} \ln \frac{d}{d E} \mathfrak{n}\left(E ; H_{\mathrm{cl}}^{(n)}\right) \\
& =\left(\frac{n d}{2}-1\right) k \frac{\int_{\Lambda_{0}^{n}} \Theta\left(E-V^{(n)}(x)\right)\left(E-V^{(n)}(x)\right)^{n d / 2-2} d^{n d} x}{\int_{\Lambda_{0}^{n}} \Theta\left(E-V^{(n)}(x)\right)\left(E-V^{(n)}(x)\right)^{n d / 2-1} d^{n d} x}, \quad n d \geq 3 .
\end{aligned}
$$

which is obviously nonnegative.

The motivation is as follows. We consider the periodic case only, the random case can be treated similarly. For a given finite volume $\Lambda$ the temperature can be defined by means of the relation $(2.2)$, i.e.,

$$
\frac{1}{T\left(E ; H_{\mathrm{cl}, \Lambda}^{(n)}\right)}=k \frac{d}{d E} \ln \frac{d N\left(E ; H_{c l, \Lambda}^{(n)}\right)}{d E}
$$

with

$$
N\left(E ; H_{\mathrm{cl}, \Lambda}^{(n)}\right)=\int_{\Lambda^{n}} \Theta\left(E-V^{(n)}(x)\right)\left(E-V^{(n)}(x)\right)^{n d / 2-1} d^{n d} x .
$$

Considering the limit $\Lambda \rightarrow \infty$ in the Fisher sense due to the relations

$$
\frac{d^{k}}{d E^{k}} \mathfrak{n}\left(E ; H_{\mathrm{cl}}^{(n)}\right)=\lim _{\Lambda \rightarrow \infty} \frac{1}{|\Lambda|^{n}} \frac{d^{k}}{d E^{k}} N\left(E ; H_{c l, \Lambda}^{(n)}\right), \quad k=1,2,
$$

we obtain $\lim _{\Lambda \rightarrow \infty} T\left(E ; H_{c l, \Lambda}^{(n)}\right)=T\left(E ; H_{\mathrm{cl}}^{(n)}\right)$.

The condition

$$
\frac{d}{d E} \mathfrak{n}\left(E ; H_{\mathrm{cl}}^{(n)}\right) \frac{d^{3}}{d E^{3}} \mathfrak{n}\left(E ; H_{\mathrm{cl}}^{(n)}\right) \leq\left(\frac{d^{2}}{d E^{2}} \mathfrak{n}\left(E ; H_{\mathrm{cl}}^{(n)}\right)\right)^{2}, \quad n d \geq 5
$$

guarantees that the temperature $T\left(E ; H_{\mathrm{cl}}^{(n)}\right)$ is a non-decreasing function of the energy, i.e., it naturally replaces condition (2.4). It may be rewritten as

$$
\left(\frac{n d}{2}-2\right)\left\langle\mathcal{T}_{E}{ }^{-2}\right\rangle_{E} \leq\left(\frac{n d}{2}-1\right)\left\langle\mathcal{T}_{E}{ }^{-1}\right\rangle_{E}^{2}
$$

Here $\mathcal{T}_{E}(x)^{-1}=(n d / 2-1)\left(E-V^{(n)}(x)\right)^{-1}$ and \langle\rangle$_{E}$ is the mean given by the probability measure

$$
d \mu_{E}(x)=\frac{\Theta\left(E-V^{(n)}(x)\right)\left(E-V^{(n)}(x)\right)^{n d / 2-1} d^{n d} x}{\int_{\Lambda_{0}^{n}} \Theta\left(E-V^{(n)}(x)\right)\left(E-V^{(n)}(x)\right)^{n d / 2-1} d^{n d} x}
$$

on $\Lambda_{0}^{n}$ provided $E$ is such that the set $\left\{x \mid V^{(n)}(x) \leq E\right\}$ has non-zero measure. Thus, we obtain the relation similar to 2.5 ,

$$
\frac{1}{T(E)}=k\left\langle\mathcal{T}_{E}^{-1}\right\rangle_{E}
$$

Note that the heat capacity will be negative if the inequality opposite to (2.27) holds. By the Schwarz inequality we have

$$
\left\langle\mathcal{T}_{E}{ }^{-2}\right\rangle_{E} \geq\left\langle\mathcal{T}_{E}{ }^{-1}\right\rangle_{E}^{2}
$$

On the other hand $(n d / 2-2)<(n d / 2-1)$. Therefore for the heat capacity to be negative the inequality 2.29) has to be sufficiently strict.

As an example where the temperature decreases with the energy consider the following choice for the periodic potential. Let $X \subset \Lambda_{0}$ have measure $0<\alpha<1$. Define $V(x)=E_{0}>0$ 
for $x \in X$ and zero otherwise when $x \in \Lambda_{0} \backslash X$. Finally extend $V$ periodically to all of $\mathbb{R}^{d}$. In what follows $E_{0}$ will be fixed. Then for any $E>n E_{0}$ and $k=1,2,3$

$$
\begin{array}{r}
0 \leq \int_{\Lambda_{0}^{n}} \Theta\left(E-V^{(n)}(x)\right)\left(E-V^{(n)}(x)\right)^{n d / 2-k} d^{n d} x \\
=\sum_{j=0}^{n}\left(\begin{array}{c}
n \\
j
\end{array}\right) \alpha^{j}(1-\alpha)^{n-j}\left(E-j E_{0}\right)^{n d / 2-k} .
\end{array}
$$

We now fix $\alpha$ to be given as $\alpha=1-\left(\left(E-n E_{0}\right) / E\right)^{(n d / 2-2) / n}$. Then the leading contribution to (2.30) when $E-n E_{0}$ is small is given as

$$
\begin{aligned}
\left(E-n E_{0}\right)^{n d / 2-3} & \text { for } \quad k=3, \\
\text { const } \cdot\left(E-n E_{0}\right)^{n d / 2-2} & \text { for } \quad k=1,2 .
\end{aligned}
$$

When inserted into (2.27) the 1.h.s. behaves to leading order as const $\cdot\left(E-n E_{0}\right)^{n d / 2-5}$, whereas the r.h.s. behaves like const $\cdot\left(E-n E_{0}\right)^{n d / 2-4}$ with positive constants depending on $E, n$ and $d$. Therefore when $E>n E_{0}$ is sufficiently close to $n E$ the inequality in (2.27) is indeed reversed. Having thus fixed $\alpha$ by continuity the heat capacity therefore also becomes negative for all energies sufficiently close to $E$. Starting from the potential just constructed by a small change we may achieve that $V$ is smooth. By the same arguments we may replace $\Lambda_{0}$ by any $\Lambda$ and find a potential $V$ supported in $\Lambda$ such that the heat capacity defined by $N\left(E ; H_{\mathrm{cl} \Lambda}^{(n)}\right)$ is negative at least in some energy interval.

By the representation (2.28) we may also define the inverse temperature fluctuation $\Delta\left(T^{-1}\right)(E)=\Delta\left(T^{-1}\right)\left(E ; H_{\mathrm{cl}}^{(n)}\right)$ by

$$
\Delta\left(T^{-1}\right)(E)^{2}=k^{2}\left(\left\langle\mathcal{T}_{E}^{-2}\right\rangle_{E}-\left\langle\mathcal{T}_{E}^{-1}\right\rangle_{E}^{2}\right) \geq 0
$$

Recalling the definition of the heat capacity as $1 / c_{\mathrm{v}}(E)=d T(E) / d E$ we obtain in the case $n d \geq 5$

$$
\frac{1}{c_{\mathrm{v}}(E)}=-k T(E)^{2}\left[\frac{\left(\frac{n d}{2}-2\right)}{\left(\frac{n d}{2}-1\right)}\left\langle\mathcal{T}_{E}{ }^{-2}\right\rangle_{E}-\left\langle\mathcal{T}_{E}^{-1}\right\rangle_{E}^{2}\right]
$$

and therefore

$$
\Delta\left(T^{-1}\right)(E)^{2}+\frac{k}{T(E)^{2} c_{\mathrm{v}}(E)}=k^{2}\left(\frac{n d}{2}-1\right)^{-1}\left\langle\mathcal{T}_{E}^{-2}\right\rangle_{E} \geq 0 .
$$

In the absence of external fields, i.e., for $V=0$ this gives $\Delta(1 / T)(E)=0$. Since this is an undesirable feature, we will consider the following alternative. Let $E_{\min }^{(n)}=\inf _{x \in \mathbb{R}^{n d}} V^{(n)}(x)>$ $-\infty$. Motivated by the construction (2.13) consider the probability measure on the interval $\left[E_{\min }^{(n)}, E\right]$

$$
d v_{E}\left(E^{\prime}\right)=\frac{1}{\frac{d}{d E} \mathfrak{n}\left(E ; H_{\mathrm{cl}}^{(n)}\right)} \frac{d^{2}}{d E^{\prime 2}} \mathfrak{n}\left(E^{\prime} ; H_{\mathrm{cl}}^{(n)}\right) d E^{\prime},
$$

such that all derivatives (provided they exist) of $\mathfrak{n}\left(E^{\prime} ; H_{\mathrm{cl}}^{(n)}\right)$ with respect to $E^{\prime}>E_{\min }^{(n)}$ are positive. Denote the resulting mean by \langle\rangle$_{E}^{\prime}$. Observe that

$$
\left.\frac{d v_{E}\left(E^{\prime}\right)}{d E^{\prime}}\right|_{E^{\prime}=E-0}=\frac{1}{k T(E)}
$$

and so again we have

$$
\frac{1}{T(E)}=k\left\langle\mathcal{T}^{-1}\right\rangle_{E}^{\prime}
$$


with

$$
\mathcal{T}\left(E^{\prime}\right)^{-1}=\frac{d}{d E^{\prime}} \ln \frac{d^{2}}{d E^{\prime 2}} \mathfrak{n}\left(E^{\prime} ; H_{\mathrm{cl}}^{(n)}\right) .
$$

In analogy to (2.14) this results in an alternative definition of the fluctuation of the inverse temperature of the form

$$
\Delta^{\prime}\left(T^{-1}\right)(E)=\left(k^{2}\left\langle\mathcal{T}^{-2}\right\rangle_{E}^{\prime}-T(E)^{-2}\right)^{1 / 2}
$$

and which in general differs from $\Delta\left(T^{-1}\right)(E)$ given by (2.31). For comparison we first observe that $(n d \geq 5)$

$$
\left\langle\mathcal{T}_{E}^{-2}\right\rangle_{E}=\frac{\left(\frac{n d}{2}-1\right)}{\left(\frac{n d}{2}-2\right)} \frac{d^{3}}{d E^{3}} \mathfrak{n}\left(E ; H_{\mathrm{cl}}^{(n)}\right) / \frac{d}{d E} \mathfrak{n}\left(E ; H_{\mathrm{cl}}^{(n)}\right) .
$$

On the other hand

$$
\left\langle\mathcal{T}^{-2}\right\rangle_{E}^{\prime}=\int_{E_{\min }^{(n)}}^{E}\left(\left(\frac{d^{3}}{d E^{\prime 3}} \mathfrak{n}\left(E^{\prime} ; H_{\mathrm{cl}}^{(n)}\right)\right)^{2} / \frac{d^{2}}{d E^{\prime 2}} \mathfrak{n}\left(E^{\prime} ; H_{\mathrm{cl}}^{(n)}\right)\right) d E^{\prime} / \frac{d}{d E} \mathfrak{n}\left(E ; H_{\mathrm{cl}}^{(n)}\right) .
$$

For $n d \geq 7$ we perform a partial integration in (2.34) and obtain by comparison

$$
\left\langle\mathcal{T}^{-2}\right\rangle_{E}^{\prime}=\frac{\left(\frac{n d}{2}-2\right)}{\left(\frac{n d}{2}-1\right)}\left\langle\mathcal{T}_{E}^{-2}\right\rangle_{E}+\langle\tilde{\mathcal{G}}\rangle_{E}^{\prime}
$$

with

$$
\tilde{\mathcal{G}}\left(E^{\prime}\right)=-\frac{d}{d E^{\prime}} \frac{1}{\mathcal{T}\left(E^{\prime}\right)} .
$$

The condition $\mathcal{G}\left(E^{\prime}\right) \geq 0$, which means that $\mathcal{T}\left(E^{\prime}\right)$ increases at $E^{\prime}$, is equivalent to

$$
\frac{d^{2}}{d E^{\prime 2}} \mathfrak{n}\left(E^{\prime} ; H_{\mathrm{cl}}^{(n)}\right) \frac{d^{4}}{d E^{\prime^{4}}} \mathfrak{n}\left(E^{\prime} ; H_{\mathrm{cl}}^{(n)}\right) \leq\left(\frac{d^{3}}{d E^{\prime^{3}}} \mathfrak{n}\left(E^{\prime} ; H_{\mathrm{cl}}^{(n)}\right)\right)^{2},
$$

which compares with $(2.26)$ and hence may be discussed in a similar way. By (2.32) and (2.35) the heat capacity can be expressed as

$$
\frac{1}{c_{\mathrm{v}}(E)}=-k^{2}\left[\left\langle\mathcal{T}^{-2}\right\rangle_{E}^{\prime}-\langle\tilde{\mathcal{G}}\rangle_{E}^{\prime}-\left\langle\mathcal{T}^{-1}\right\rangle_{E}^{\prime 2}\right]
$$

This alternative definition of the fluctuation and the heat capacity are therefore related by

$$
\Delta^{\prime}\left(T^{-1}\right)(E)^{2}+\frac{k}{T(E)^{2} c_{\mathrm{v}}(E)}=k T(E)^{2}\langle\tilde{\mathcal{G}}\rangle_{E}^{\prime} .
$$

As a test in the special case $V=0$, i.e., in the absence of external fields, and for $n d>6$ this new fluctuation is calculated explicitly to be

$$
\Delta^{\prime}\left(T^{-1}\right)(E)=\left(\frac{n d}{2}-3\right)^{-1 / 2}\left(\frac{n d}{2}-1\right)^{-1 / 2} \frac{1}{T(E)} .
$$

If we define the temperature fluctuation as $\Delta^{\prime}(T)(E)=\Delta^{\prime}\left(T^{-1}\right)(E) T(E)^{2}$ then for large $n d$ we obtain $\Delta^{\prime}(T)(E) \approx(2 / n d) T(E)$, now as it should be.

The following asymptotic expansion holds for large $E$ and $n d>2$

$$
\begin{aligned}
\frac{\Gamma\left(\frac{n d}{2}+1\right)}{(2 \pi m)^{n d / 2}} \frac{d}{d E} \mathfrak{n}\left(E ; H_{\mathrm{cl}}^{(n)}\right) & =\frac{n d}{2} \int_{\Lambda_{0}^{n}} \Theta\left(E-V^{(n)}(x)\right)\left(E-V^{(n)}(x)\right)^{n d / 2-1} d^{n d} x \\
& =\frac{n d}{2} E^{n d / 2-1}\left[1-a E^{-1}+b E^{-2}+O\left(E^{-3}\right)\right],
\end{aligned}
$$


where

$$
\begin{aligned}
a & =\left(\frac{n d}{2}-1\right) \int_{\Lambda_{0}^{n}} V^{(n)}(x) d^{n d} x=n\left(\frac{n d}{2}-1\right) \bar{V}, \\
b & =\frac{1}{2}\left(\frac{n d}{2}-1\right)\left(\frac{n d}{2}-2\right) \int_{\Lambda_{0}^{n}}\left(V^{(n)}(x)\right)^{2} d^{n d} x \\
& =\frac{1}{2}\left(\frac{n d}{2}-2\right)\left(\frac{n d}{2}-1\right)\left[n \overline{V^{2}}+n(n-1) \bar{V}^{2}\right] .
\end{aligned}
$$

Here we use the following notation. In the periodic case and for any natural number $k$

$$
\overline{V^{k}}=\int_{\Lambda_{0}} V(x)^{k} d^{d} x=\int_{\Lambda_{0}} V_{0}(x)^{k} d^{d} x
$$

In the stochastic case

$$
\overline{V^{k}}=\overline{q^{k}} \int_{\Lambda_{0}} V_{0}(x)^{k} d^{d} x \text { with } \overline{q^{k}}=\int q^{k} d \mu(q) .
$$

Note that $\bar{V}^{2} \leq \overline{V^{2}}$ by the Schwarz inequality with equality if and only if $V$ is constant in $x$ (and in $\omega$ in the random case). This gives the following asymptotic energy-temperature relation

$$
\begin{aligned}
\frac{1}{T\left(E ; H_{\mathrm{cl}}^{(n)}\right)}= & k\left(\frac{n d}{2}-1\right) E^{-1} \\
& \cdot\left(1+E^{-1} n \bar{V}+E^{-2} n\left[\left(n+\frac{n d}{2}-2\right) \bar{V}^{2}-\left(\frac{n d}{2}-2\right) \overline{V^{2}}\right]+O\left(E^{-3}\right)\right) .
\end{aligned}
$$

Therefore for all sufficiently large $E$ and for $n d>2$ the temperature increases with $E$. Also when $n d>2$ at fixed large energy $E$ to leading order in $E^{-1}$ the temperature $T(E)$ decreases (as a function of the potential) when a potential with $\bar{V}>0$ is switched on. Otherwise it decreases. Similarly if $\bar{V}=0$ such that $V$ is not a constant, then $T(E)$ decreases for all large $n d$ when such a potential is switched on.

For the heat capacity we obtain the asymptotic expansion for large energies

$$
c_{\mathrm{v}}\left(E ; H_{\mathrm{cl}}^{(n)}\right)=k\left(\frac{n d}{2}-1\right)\left(1+E^{-2} n\left(\frac{n d}{2}-2\right)\left[\overline{V^{2}}-\bar{V}^{2}\right]+O\left(E^{-3}\right)\right) .
$$

Thus for large $E$ and $n d>4$ the heat capacity increases when a potential is switched on.

A similar discussion for a canonical ensemble is possible by defining the partition function as the Laplace-Stieltjes transform of the integrated density of states

$$
\begin{aligned}
z\left(\beta ; H_{\mathrm{cl}}^{(n)}\right) & =\int e^{-\beta E} d \mathfrak{n}\left(E ; H_{\mathrm{cl}}^{(n)}\right)=\beta \int e^{-\beta E} \mathfrak{n}\left(E ; H_{\mathrm{cl}}^{(n)}\right) d E \\
& =\lim _{\Lambda \rightarrow \infty} \frac{1}{|\Lambda|^{n}} \int e^{-\beta E} d N\left(E ; H_{c l, \Lambda}^{(n)}\right),
\end{aligned}
$$

such that $z\left(\beta ; H_{0 \mathrm{cl}}^{(n)}\right)=(2 \pi m k T)^{n d / 2}$. This limit has not to be confused with the notion of "thermodynamic limit" used when discussing extensive systems in statistical mechanics. There one divides the logarithm of the partition function in a finite volume by the volume of the configuration space and then takes the limit (see, e.g., [66]). So in order to distinguish these two limits we use the notion "per unit interaction volume" (see, however, the discussion of the quantum mechanical grand canonical ensemble in Section B). The reason for this difference is as follows. The thermodynamic limit has to be taken when the number 
of particles increases with $\Lambda$ such that the density remains constant. Here the system is not extensive since the number of particles stays fixed.

The resulting entropy, mean energy and the Helmholtz free energy are then given as

$$
\begin{aligned}
& s\left(\beta ; H_{\mathrm{cl}}^{(n)}\right)=-k \beta^{2} \frac{d}{d \beta}\left(\frac{1}{\beta} z\left(\beta ; H_{\mathrm{cl}}^{(n)}\right)\right), \\
& \bar{e}\left(\beta ; H_{\mathrm{cl}}^{(n)}\right)=-\frac{d}{d \beta} \ln z\left(\beta ; H_{\mathrm{cl}}^{(n)}\right), \\
& f\left(\beta ; H_{\mathrm{cl}}^{(n)}\right)=-\frac{1}{\beta} \ln z\left(\beta ; H_{\mathrm{cl}}^{(n)}\right),
\end{aligned}
$$

giving the familiar relation between these three quantities

$$
\bar{e}\left(\beta ; H_{\mathrm{cl}}^{(n)}\right)=T s\left(\beta ; H_{\mathrm{cl}}^{(n)}\right)+f\left(\beta ; H_{\mathrm{cl}}^{(n)}\right) .
$$

In analogy to 2.16 the heat capacity in this canonical setup is given as $c_{\mathrm{v}}\left(T ; H_{\mathrm{cl}}^{(n)}\right)=$ $d \bar{e}\left(\beta ; H_{\mathrm{cl}}^{(n)}\right) / d T$.

Also in analogy to the discussion following (2.17) we may relate the microcanonical and the canonical description. We will assume $n d>5$ such that $\mathfrak{n}\left(E ; H_{\mathrm{cl}}^{(n)}\right)$ is three times differentiable w.r.t. $E$. Write

$$
z\left(\beta ; H_{\mathrm{cl}}^{(n)}\right)=\int e^{-\beta E} \frac{d}{d E} \mathfrak{n}\left(E ; H_{\mathrm{cl}}^{(n)}\right) d E=\beta \int e^{-\beta E+\ln \left(\frac{1}{\beta} \frac{d}{d E} \mathfrak{n}\left(E ; H_{\mathrm{cl}}^{(n)}\right)\right)} d E .
$$

Then the integrand takes its extremal value at $E_{\max }=E_{\max }(T)$ given implicitly by

$$
\frac{1}{T}=\left.k \frac{d}{d E} \ln \frac{d}{d E} \mathfrak{n}\left(E ; H_{\mathrm{cl}}^{(n)}\right)\right|_{E=E_{\max }}
$$

Relation (2.45) now compares with 2.25). In other words the temperature defined in the microcanonical ensemble at the energy $E=E_{\max }$ agrees with the chosen one for the canonical ensemble. If a solution $E_{\max }$ to this relation exists it is unique and a maximum provided the inequality (2.27) holds for all $E$ (for all large $E$ this is true by (2.42)) and is a strict inequality at $E=E_{\max }$. Indeed, for $E$ near $E_{\max }$ we obtain

$$
-\beta E+\ln \left(\frac{1}{\beta} \frac{d}{d E} \mathfrak{n}\left(E ; H_{\mathrm{cl}}^{(n)}\right)\right)=-\frac{1}{2} \alpha\left(E-E_{\max }\right)^{2}+O\left(\left(E-E_{\max }\right)^{3}\right)
$$

with $\alpha=\alpha\left(\beta ; H_{\mathrm{cl}}^{(n)}\right)$ now given by

$$
\alpha=-\left.\frac{d^{2}}{d E^{2}} \ln \frac{d}{d E} \mathfrak{n}\left(E ; H_{\mathrm{cl}}^{(n)}\right)\right|_{E=E_{\max }},
$$

which is nonnegative if 2.27 ) holds and is positive if $(2.27)$ is a strict inequality at $E=E_{\max }$. Again as in the discussion after (2.17) and in the microcanonical notation, by (2.45) $\alpha$ is related to the heat capacity via $\alpha=1 /\left(k c_{\mathrm{v}}\left(E_{\max }\right) T\left(E_{\max }\right)^{2}\right)$.

Hence to a good approximation and for given temperature the canonical ensemble is described in terms of a Gaussian distribution in energy with variance $\alpha^{-1 / 2}$ in case $\alpha$ is positive and large. In particular in this approximation we obtain $\bar{e}\left(\beta ; H_{\mathrm{cl}}^{(n)}\right) \approx E_{\max }$ and $\Delta \bar{e}\left(\beta ; H_{\mathrm{cl}}^{(n)}\right) \approx \alpha^{-1 / 2}$. Similarly the canonical heat capacity and the microcanonical heat capacity at energy $E_{\max }$ agree in this approximation (see the discussion after (2.19)). Observe that for large $T$ the implicit equation (2.45) has always at least one solution $E_{\max } \approx$ $(n d / 2-1) k T$ due to 2.38), which by (2.42) is at least a local maximum. In the free case by 2.45) $E_{\max }=(n d / 2-1) k T$ (which compares with the energy-temperature relation $E=(n d / 2-1) T(E)$ in the microcanonical ensemble for the free case $)$ and $\alpha^{-1}=(n d / 2-$ $1)^{-1} E_{\max }^{2}=(n d / 2-1)(k T)^{2}$. So as a function of the particle number $n$ in the free case the 
variance $\alpha^{-1 / 2}$ goes like $n^{-1 / 2}$ when the energy is fixed and like $n^{1 / 2}$ when the temperature is fixed. We expect this feature to extend to the general case. This would in particular imply that at fixed given energy the difference between the microcanonical and the canonical description decreases with increasing particle number. small $\beta$

For periodic and bounded potentials the following asymptotic expansion holds for

$$
z\left(\beta ; H_{\mathrm{cl}}^{(n)}\right)=z\left(\beta ; H_{0 \mathrm{cl}}^{(n)}\right)\left(1-\beta n \bar{V}+\frac{\beta^{2}}{2}\left[n \overline{V^{2}}+n(n-1) \bar{V}^{2}\right]+O\left(\beta^{3}\right)\right) .
$$

Note that the Laplace transform of the asymptotic expansion (2.38) gives this asymptotic expansion. Relation (2.46) results in the following asymptotic temperature-energy relation

$$
\bar{e}\left(\beta ; H_{\mathrm{cl}}^{(n)}\right)=\frac{n d}{2} \beta^{-1}+n \bar{V}-\beta n\left[\overline{V^{2}}-\bar{V}^{2}\right]+O\left(\beta^{2}\right) .
$$

So for fixed small $\beta$ to leading order the mean energy increases when a potential with $\bar{V}>0$ is switched on. Otherwise it decreases. This behavior is in agreement with the corresponding result (2.41) in the microcanonical description. If $\bar{V}=0$ then the mean energy increases when such a potential is switched on.

For large $E$ and $T$ respectively the comparison between the microcanonical and the canonical description can be made more explicit. Indeed, relation 2.47 may be inverted to give

$$
\begin{aligned}
\frac{1}{T}= & k \frac{n d}{2} \bar{e}\left(\beta ; H_{\mathrm{cl}}^{(n)}\right)^{-1}\left(1+\bar{e}\left(\beta ; H_{\mathrm{cl}}^{(n)}\right)^{-1} n \bar{V}\right. \\
& \left.+\bar{e}\left(\beta ; H_{\mathrm{cl}}^{(n)}\right)^{-2} n\left[\left(n+\frac{n d}{2}\right) \bar{V}^{2}-\frac{n d}{2} \overline{V^{2}}\right]+O\left(\bar{e}\left(\beta ; H_{\mathrm{cl}}^{(n)}\right)^{-3}\right)\right)
\end{aligned}
$$

which compares with the microcanonical relation 2.41). In other words for $E$ and $n$ large the temperature defined in the microcanonical ensemble agrees with the one chosen for the canonical ensemble, i.e., we have

$$
T \approx T\left(\bar{e}\left(\beta=1 / k T ; H_{\mathrm{cl}}^{(n)}\right)\right), \quad E \approx \bar{e}\left(\beta=1 / k T\left(E ; H_{\mathrm{cl}}^{(n)}\right) ; H_{\mathrm{cl}}^{(n)}\right) .
$$

From 2.47) we obtain the asymptotic expansion for small $\beta$

$$
c_{\mathrm{v}}\left(\beta ; H_{\mathrm{cl}}^{(n)}\right)=\frac{k n d}{2}+\frac{k n \beta^{2}}{2}\left[\overline{V^{2}}-\bar{V}^{2}\right]+O\left(\beta^{3}\right) .
$$

So if a potential is switched on, the heat capacity increases when $\beta$ is small. Setting

$$
\Delta \bar{e}\left(\beta ; H_{\mathrm{cl}}^{(n)}\right)=\left(\frac{d^{2}}{d \beta^{2}} \ln z\left(\beta ; H_{\mathrm{cl}}^{(n)}\right)\right)^{1 / 2}=\left(k T^{2} c_{\mathrm{v}}\left(T ; H_{\mathrm{cl}}^{(n)}\right)\right)^{1 / 2}
$$

to be the energy fluctuation we obtain for its asymptotic behavior

$$
\Delta \bar{e}\left(\beta ; H_{\mathrm{cl}}^{(n)}\right)=\sqrt{\frac{n d}{2}} \frac{1}{\beta}\left(1+\frac{\beta^{2}}{d}\left[\overline{V^{2}}-\bar{V}^{2}\right]+O\left(\beta^{3}\right)\right) .
$$

\section{The quantum theory}

In analogy to the classical case the microcanonical ensemble for a quantum system is usually given as follows. Let $\{\mathcal{H}, H\}$ be a quantum mechanical system, where $\mathcal{H}$ is a 
Hilbert space and $H$ a Hamilton operator on $\mathcal{H}$ with a purely discrete spectrum. Motivated by the classical case on might be tempted to define the the following microcanonical quantity

$$
\frac{d}{d E} N(E ; H)=\frac{d}{d E} \operatorname{tr}_{\mathcal{H}}(\Theta(E-H))=\operatorname{tr}_{\mathcal{H}}(\delta(E-H)) .
$$

As is well known (see, e.g., [35]) this procedure encounters difficulties for the following reason. Since $H$ has a purely discrete spectrum $N(E ; H)=\operatorname{tr}_{\mathcal{H}}(\Theta(E-H))$ is given as the number of eigenvalues (counting multiplicities) of $H$ up to energy $E$. But then $d N(E ; H) / d E$ is a sum of $\delta$-functions at the eigenvalues of $H$ with coefficients given by the multiplicities of these eigenvalues. In other words $d N(E ; H) / d E$ is a generalized function. On the other hand if $H$ has continuous spectrum then both $\operatorname{tr}_{\mathcal{H}}(\Theta(E-H))$ and $\operatorname{tr}_{\mathcal{H}}(\delta(E-H))$ do not make sense. This is in our point of view the main obstacle in making the microcanonical concept useful in the quantum context. The usual way out is to consider "large systems", where the spacing of the eigenvalues becomes small and to replace $d N(E ; H) / d E$ by $\operatorname{tr}_{\mathcal{H}}(\Theta(E+\Delta E-H)-\Theta(E-H))$ with $\Delta E$ being small. This, however, leads to another dilemma, since usually there is no natural and intrinsic choice for the size of $\Delta E$.

The approach we will propose will give a precise meaning to the notion "large system" in the sense that the number of particles $n$ only should be sufficiently large. The oneparticle operators $H=H^{(1)}$ we will consider are supposed to have continuous spectrum. More precisely, we will consider one-particle Schrödinger operators acting on the Hilbert space $L^{2}\left(\mathbb{R}^{d}\right)$ and of the form

$$
H=H_{0}+V,
$$

Here $H_{0}$ is the free Hamiltonian for a particle of mass $m>0$

$$
H_{0}=-\frac{\hbar^{2}}{2 m} \Delta
$$

where $\Delta$ is the Laplace operator on $\mathbb{R}^{d}$, the configuration space for the particle. The potentials $V$ we have in mind are periodic or random as in (2.20).

The resulting $n$-particle operators with no interaction between the particles will be denoted by $H^{(n)}$. They act on the $n$-fold tensor product $\otimes_{n} L^{2}\left(\mathbb{R}^{d}\right)$ of $L^{2}\left(\mathbb{R}^{d}\right)$, which is equal to $L^{2}\left(\mathbb{R}^{n d}\right)$, if we consider the particles to be distinguishable or on the subspaces $\otimes_{n, \pm} L^{2}\left(\mathbb{R}^{d}\right)$ of symmetric (+) or antisymmetric (-) wave functions, when we allow for statistics.

Let $\chi_{\Lambda}$ be the characteristic function of any cube $\Lambda \subset \mathbb{R}^{d}$ and correspondingly let $\chi_{\Lambda^{n}}$ denote the characteristic function of $\Lambda^{n} \subset \mathbb{R}^{n d}$. Set $V_{\Lambda}=\chi_{\Lambda} V$. Also let $\Delta_{\Lambda}$ be the Laplace operator on $\Lambda$ with Dirichlet or Neumann boundary conditions. Let $H_{\Lambda}=H_{0, \Lambda}+V_{\Lambda}$ with $H_{0, \Lambda}=-\hbar^{2} / 2 m \cdot \Delta_{\Lambda}$ and correspondingly $H_{\Lambda}^{(n)}$ for the resulting $n$-particle operator. In the examples we have in mind all $H_{\Lambda}^{(n)}$ have a discrete spectrum with no finite accumulation points such that $N\left(E ; H_{\Lambda}^{(n)}\right)$ as well as the canonical Boltzmann-Gibbs partition function is well defined. Furthermore it can be shown that the limit, called the integrated density of states,

$$
0 \leq \mathfrak{n}\left(E ; H^{(n)}\right)=\lim _{\Lambda \rightarrow \infty} \frac{1}{|\Lambda|^{n}} N\left(E ; H_{\Lambda}^{(n)}\right)
$$

exists [61] and is independent of the boundary conditions [56]. In particular in the free $n$-particle case $\mathfrak{n}\left(E ; H_{0}^{(n)}\right)=0$ for $E<0$

$$
\mathfrak{n}\left(E ; H_{0}^{(n)}\right)=\frac{1}{\Gamma\left(\frac{n d}{2}+1\right)}\left(\frac{m E}{2 \pi \hbar^{2}}\right)^{n d / 2}=\frac{(2 \pi m E)^{n d / 2}}{h^{n d} \Gamma\left(\frac{n d}{2}+1\right)}
$$


for $E>0$. This reflects the well known intuitive observation that each quantum state occupies the volume $h^{n}(h=2 \pi \hbar)$ in the phase space $\mathbb{R}^{2 n d}$. In other words a comparison with the classical case (2.22) gives the relation $h^{\text {nd }} \mathfrak{n}\left(E ; H_{0}^{(n)}\right)=\mathfrak{n}\left(E ; H_{0 \mathrm{cl}}^{(n)}\right)$. So also $\mathfrak{n}\left(E ; H_{0}^{(n)}\right)$ is smooth for $E \neq 0$ and $[n d / 2]$ times differentiable at $E=0$ (here $[a]$ denotes integer part of $a$ ). The smoothness of $\mathfrak{n}\left(E ; H_{0}^{(n)}\right)$, i.e., the order of differentiability, increases with the number of particles.

For random potentials, i.e., when we consider $H=H_{\omega}=H_{0}+V_{\omega}$, the integrated density of states $\mathfrak{n}\left(E ; H_{\omega}\right)$ is actually a deterministic quantity, i.e., independent of $\omega$ for almost all $\omega$. The same is also true for the spectrum $\operatorname{spec}(H)$ of $H_{\omega}$ and in particular for its infimum inf $\operatorname{spec}\left(H_{\omega}\right)$ [61]. This will have the important consequence that all other quantities we will introduce using the integrated density of states will also be deterministic. So in order to cover both periodic and random potentials simultaneously we will simply write $\mathfrak{n}(E ; H)$ for $\mathfrak{n}\left(E ; H_{\omega}\right)$ and similarly $\mathfrak{n}\left(E ; H^{(n)}\right)$ for the $n$-particle case.

Obviously $\mathfrak{n}\left(E ; H^{(n)}\right)$ is a monotone increasing function in $E$ and therefore continuous at almost all $E$. Its derivative, if it exists, is called the density of states for $H^{(n)}$. Also by construction $\mathfrak{n}\left(E+c ; H^{(n)}+c\right)=\mathfrak{n}\left(E ; H^{(n)}\right)$ for any constant $c$ and $n\left(\lambda E ; \lambda H^{(n)}\right)=\mathfrak{n}\left(E ; H^{(n)}\right)$ for all $\lambda>0$. Note also that in general $\mathfrak{n}\left(E ; H^{(n)}\right)$ need not vanish for $E<0$.

If the potential is sign-definite, i.e., $\pm V \geq 0$ such that $\pm V_{\Lambda} \geq 0$ for all $\Lambda$ then by the min-max principle (see, e.g., [14]) we have $\mp N\left(E ; H_{\Lambda}^{(n)}\right) \geq \mp N\left(E ; H_{0, \Lambda}\right)$ for all $\Lambda$ and $E$ and $n$. This gives

$$
\mp \mathfrak{n}\left(E ; H^{(n)}\right) \geq \mp \mathfrak{n}\left(E ; H_{0}^{(n)}\right) \text { for all E if } \pm V \geq 0
$$

and more generally

$$
\mathfrak{n}\left(E ;\left(H_{0}+V_{1}\right)^{(n)}\right) \leq \mathfrak{n}\left(E ;\left(H_{0}+V_{2}\right)^{(n)}\right) \text { for all } \mathrm{E} \text { if } V_{1} \geq V_{2} .
$$

Alternatively the integrated density of states may be written in the form

$$
\mathfrak{n}\left(E ; H^{(n)}\right)=\lim _{\Lambda \rightarrow \infty} \frac{1}{|\Lambda|^{n}} \operatorname{tr}_{L^{2}\left(\mathbb{R}^{n d}\right)}\left(\chi_{\Lambda^{n}} \Theta\left(E-H^{(n)}\right)\right) .
$$

Therefore, for periodic $V$

$$
\mathfrak{n}\left(E ; H^{(n)}\right)=\operatorname{tr}_{L^{2}\left(\mathbb{R}^{n d}\right)}\left(\chi_{\Lambda_{0}^{n} \Theta} \Theta\left(E-H^{(n)}\right)\right),
$$

where $\Lambda_{0}$ is the unit cube in $\mathbb{R}^{d}$. This relation also continues to hold for random operators, when the right hand side is replaced by its average over the random variable. That the integrated density of states is actually deterministic is due to the limit relation in (3.5), which is called a self-averaging effect. Below we will show how to obtain $\mathfrak{n}\left(E ; H^{(n)}\right)$ for all particle numbers $n$ from the one particle integrated density of states $\mathfrak{n}(E ; H)$.

One-particle scattering theory may now be used to relate $\mathfrak{n}\left(E ; H_{0}+V\right)$ and $\mathfrak{n}\left(E ; H_{0}\right)$. More precisely let $\delta\left(E ; H_{0}+V_{\Lambda}, H_{0}\right)$ denote the phase shift at energy $E>0$ for the pair $\left(H_{0}+V_{\Lambda}, H_{0}\right)$. In other words, if $S\left(E ; H_{0}+V_{\Lambda}, H_{0}\right)$ is the scattering matrix for the pair $\left(H_{0}+V_{\Lambda}, H_{0}\right)$ at energy $E$ then $\operatorname{det} S\left(E ; H_{0}+V_{\Lambda}, H_{0}\right)=\exp \left(2 i \delta\left(E ; H_{0}+V_{\Lambda}, H_{0}\right)\right)$. For $E>0$ one has [45, 46]

$$
\mathfrak{n}(E ; H)-\mathfrak{n}\left(E ; H_{0}\right)=\lim _{\Lambda \rightarrow \infty} \frac{1}{\pi|\Lambda|} \delta\left(E ; H_{0}+V_{\Lambda}, H_{0}\right)=\frac{1}{\pi} \hat{\delta}\left(E ; H, H_{0}\right) .
$$

Now $-\delta\left(E ; H_{0}+V_{\Lambda}, H_{0}\right) / \pi$ may be replaced by the so-called spectral shift function $\xi\left(E ; H_{0}+\right.$ $V_{\Lambda}, H_{0}$ ) of Krein (see, e.g., [8]) which for negative $E$ equals minus the number of bound states below $E$. We use the spectral shift function to extend the scattering phase to all energies in such a way that $\delta\left(E ; H_{0}+V_{\Lambda}, H_{0}\right)=-\pi \xi\left(E ; H_{0}+V_{\Lambda}, H_{0}\right)$. With this extension of 
$\delta\left(E ; H_{0}+V_{\Lambda}, H_{0}\right)$ to all $E$ relation (3.7) also extends to all $E$. In particular, for random potentials $\hat{\delta}\left(E ; H, H_{0}\right)$ is deterministic and $\mathfrak{n}(E ; H)=\hat{\delta}\left(E ; H, H_{0}\right)=0$ for $E<\min (\inf \operatorname{spec}(H), 0)$.

The trace formula for the spectral shift function leads to the following relation which is due to Beth and Uhlenbeck 汭,

$$
\operatorname{tr}_{L^{2}\left(\mathbb{R}^{d}\right)}\left(e^{-\beta\left(H_{0}+V_{\Lambda}\right)}-e^{-\beta H_{0}}\right)=\frac{\beta}{\pi} \int e^{-\beta E} \delta\left(E ; H_{0}+V_{\Lambda}, H_{0}\right) d E, \quad \beta>0 .
$$

The spectral shift density $\hat{\delta}\left(E ; H, H_{0}\right)$ in (3.7) may be interpreted as the phase shift at energy $E$ per unit interaction volume. In short we will call it the (total) scattering phase density. Also (3.7) extends to the $n$-particle case giving

$$
\mathfrak{n}\left(E ; H^{(n)}\right)-\mathfrak{n}\left(E ; H_{0}^{(n)}\right)=\lim _{\Lambda \rightarrow \infty} \frac{1}{\pi|\Lambda|^{n}} \delta\left(E ; H_{0}^{(n)}+V_{\Lambda^{n}}^{(n)}, H_{0}^{(n)}\right)=\frac{1}{\pi} \hat{\delta}\left(E ; H^{(n)}, H_{0}^{(n)}\right) .
$$

Here we have set $V_{\Lambda^{n}}^{(n)}(x)=\chi_{\Lambda^{n}}(x) V^{(n)}(x)$ in analogy to the definition of $V_{\Lambda}$. Also $\mathfrak{n}\left(E ; H^{(n)}\right)=$ $\hat{\delta}\left(E ; H^{(n)}, H_{0}^{(n)}\right)=0$ for $E<n \min (\inf \operatorname{spec}(H), 0)$.

A precursor to $\sqrt{3.7}$ ) due to Friedel [20, 21] (see also [4, 18, 59, 55]) in the context of a single impurity is of course well known in solid state physics. To the best of our knowledge relation (3.7) and its extension (3.9) to the integrated density of states seems to be new. Note that in case of two impurities described by two potentials $W_{1}$ and $W_{2}$, say of finite range and even non-overlapping, one has $\delta\left(E ; H_{0}+W_{1}+W_{2}, H_{0}\right) \neq \delta\left(E ; H_{0}+\right.$ $\left.W_{1}, H_{0}\right)+\delta\left(E ; H_{0}+W_{2}, H_{0}\right)$. Therefore the contribution from several impurities is not simply the sum of the contributions from the individual impurities. However, contributions from different impurities are asymptotically additive, when the distance between them becomes large [43, 44].

Relations (3.7) and (3.8) are in accordance with the well known chain rule for the phase shift

$$
\delta\left(E ; H_{1}, H_{2}\right)+\delta\left(E ; H_{2}, H_{3}\right)=\delta\left(E ; H_{1}, H_{3}\right),
$$

valid for any triple of Hamilton operators for which the scattering phase for any of the pairs exist. In particular, the relation (3.10) implies $\delta\left(E ; H_{1}, H_{2}\right)=-\delta\left(E ; H_{2}, H_{1}\right)$. Also the equalities

$$
\delta\left(\lambda E ; \lambda H_{1}, \lambda H_{2}\right)=\delta\left(E+c ; H_{1}+c, H_{2}+c\right)=\delta\left(E ; H_{1}, H_{2}\right)
$$

hold for any $\lambda>0$ and any real $c$. Relations (3.11) obviously extend to $\hat{\delta}\left(E ; H, H_{0}\right)$. The monotonicity property $(3.3)$ is related to the well known relation for the scattering phase

$$
\mp \delta\left(E ; H_{0}+V_{\Lambda}, H_{0}\right) \geq 0 \text { for all } \mathrm{E} \text { if } \pm V \geq 0,
$$

and more generally (compare (3.4))

$$
\delta\left(E ; H_{0}+V_{1, \Lambda}, H_{0}\right) \leq \delta\left(E ; H_{0}+V_{2, \Lambda}, H_{0}\right) \text { for all } \mathrm{E} \text { if } V_{1} \geq V_{2} .
$$

If $V$ has compact support, then $\delta\left(E ; H_{0}+V_{\Lambda}, H_{0}\right)$ does not depend on $\Lambda$ for all large $\Lambda$ and hence in that case $\mathfrak{n}(E ; H)=\mathfrak{n}\left(E ; H_{0}\right)$. Below (see (3.37)) we will see that this implies $\mathfrak{n}\left(E ; H^{(n)}\right)=\mathfrak{n}\left(E ; H_{0}^{(n)}\right)$ for all $n$. As mentioned in the introduction it would be interesting to obtain information from $n$-particle scattering theory on $\mathfrak{n}\left(E ; H_{0}^{(n)}+V^{(n)}+W^{(n)}\right)$ where the $n$-particle interaction $W^{(n)}$ is given in terms of two-particle interaction potentials.

From the discussion so far it is clear that the quantity $\mathfrak{n}\left(E ; H^{(n)}\right)$ is a nice quantum candidate to replace the distribution $\operatorname{tr}_{\mathcal{H}} \Theta(E-H)$ which replaces the classical distribution 
$N\left(E ; H_{\mathrm{cl}}\right)$. In particular if $\mathfrak{n}\left(E ; H^{(n)}\right)$ is differentiable such that necessarily $d \mathfrak{n}\left(E ; H^{(n)}\right) / d E \geq$ 0 then we may define

$$
s\left(E ; H^{(n)}\right)=k \ln \frac{d \mathfrak{n}\left(E ; H^{(n)}\right)}{d E}
$$

to be the entropy per unit (interaction) volume for a system of $n$ particles. We hasten to point out that this definition of the entropy needs a choice of an energy unit since $d \mathfrak{n}\left(E ; H^{(n)}\right) / d E$ has the dimension of an inverse energy. In other words, $s\left(E ; H^{(n)}\right)$ is only well defined up to a constant. One way out is to renormalize the entropy additively by choosing a given energy $E_{0}$ and to replace $s\left(E ; H^{(n)}\right)$ by $s\left(E ; H^{(n)}\right)-s\left(E_{0} ; H^{(n)}\right)$. Another way is to consider a relative entropy (see, e.g., [76] for a general discussion) say for the pair $\left(H^{(n)}, H_{0}^{(n)}\right)$ as $s\left(E ; H^{(n)}, H_{0}^{(n)}\right)=s\left(E ; H^{(n)}\right)-s\left(E ; H_{0}^{(n)}\right)$.

In case $\mathfrak{n}\left(E ; H^{(n)}\right)$ is even twice differentiable w.r.t. $E$ a temperature $T(E)=T\left(E ; H^{(n)}\right)$ may be defined by

$$
\frac{1}{T(E)}=\frac{d}{d E} s\left(E ; H^{(n)}\right)=k \frac{d^{2} \mathfrak{n}\left(E ; H^{(n)}\right) / d^{2} E}{d \mathfrak{n}\left(E ; H^{(n)}\right) / d E}
$$

which is independent of the choice of the energy unit. Obviously $T(E)$ is positive if the second derivative of $\mathfrak{n}\left(E ; H^{(n)}\right)$ is positive and below we will show that this is indeed the case for the Hamiltonians we consider if we restrict the particle number $n$ to be $\geq 4$.

In analogy to the classical case (see (2.4)) the temperature $T(E)$ defined by (3.13) is a monotone increasing function at $E$ if $\mathfrak{n}\left(E ; H^{(n)}\right)$ is three times differentiable and satisfies the inequality

$$
\frac{d^{3}}{d E^{3}} \mathfrak{n}\left(E ; H^{(n)}\right) \cdot \frac{d}{d E} \mathfrak{n}\left(E ; H^{(n)}\right) \leq\left(\frac{d^{2}}{d E^{2}} \mathfrak{n}\left(E ; H^{(n)}\right)\right)^{2} .
$$

In this case the heat capacity $c_{\mathrm{v}}(E)$ defined again by $1 / c_{\mathrm{v}}(E)=d T(E) / d E$ in this quantum mechanical, microcanonical context is positive. In the opposite case, i.e., with the inequality reversed, it will be negative.

As announced in the previous section the fluctuation of the inverse temperature may now be introduced in an analogous way by replacing $\mathfrak{n}\left(E ; H_{\mathrm{cl}}^{(n)}\right)$ by $\mathfrak{n}\left(E ; H^{(n)}\right)$. For this to work we have to assume that the particle number $n$ is so large that $\mathfrak{n}\left(E ; H^{(n)}\right)$ is three times differentiable. Below we will show that at least for periodic potentials all derivatives up to order 3 are then nonnegative.

Assume that for given $E>\inf \operatorname{spec}\left(H^{(n)}\right)$ we have strict inequality $d \mathfrak{n}\left(E ; H^{(n)}\right) / d E>0$. Define the probability measure

$$
d v_{E}\left(E^{\prime}\right)=\frac{1}{d \mathfrak{n}\left(E ; H^{(n)}\right) / d E} \frac{d^{2} \mathfrak{n}\left(E^{\prime} ; H^{(n)}\right)}{d E^{\prime 2}} d E^{\prime}
$$

on the interval $\left(\inf \operatorname{spec}\left(H^{(n)}\right), E\right]$. Note the close analogy with the classical case 2.33). Continuing this analogy we introduce the function

$$
0 \leq \frac{1}{\mathcal{T}\left(E^{\prime}\right)}=\frac{d}{d E^{\prime}} \ln \frac{d^{2}}{d E^{\prime 2}} \mathfrak{n}\left(E^{\prime} ; H^{(n)}\right) \leq \infty .
$$

With $\langle\cdot\rangle_{E}^{\prime}$ denoting the resulting mean w.r.t. $d v_{E}$ we have $T(E)^{-1}=k\left\langle\mathcal{T}^{-1}\right\rangle_{E}^{\prime}$. So again an inverse temperature fluctuation is given by

$$
\Delta^{\prime}\left(T^{-1}\right)(E)=\left(k^{2}\left\langle\mathcal{T}^{-2}\right\rangle_{E}^{\prime}-T(E)^{-2}\right)^{1 / 2} .
$$

The relation 2.36) involving this fluctuation and the heat capacity again carries over by simply replacing $\mathfrak{n}\left(E ; H_{\mathrm{cl}}^{(n)}\right)$ by $\mathfrak{n}\left(E ; H^{(n)}\right)$. 
As a first example we consider the free case $H_{0}^{(n)}$ (with or without statistics) and for which the microcanonical entropy (3.12) becomes negative for small $E>0$ and $n d>2$ by (3.2). As in the classical case by (3.2) and (3.13) the resulting temperature is related to the energy by

$$
E=\left(\frac{n d}{2}-1\right) k T(E)
$$

For $n d$ large this gives for the energy per particle $E / n \approx d / 2 k T$ as should be expected. Again as in the classical case only when $n=d=1$ the temperature so defined becomes negative. For $n d>6$ the inverse temperature fluctuation is easily calculated to agree with the corresponding result $(2.37)$ in the classical case.

The next relation gives the temperature difference between that of the theory with a potential and that of the free theory at the same energy involving the potential only through the scattering phase density,

$$
T\left(E ; H^{(n)}\right)-T\left(E ; H_{0}^{(n)}\right)=\frac{1}{k} \frac{\frac{d}{d E} \hat{\delta}\left(E ; H^{(n)}, H_{0}^{(n)}\right)-E \frac{d^{2}}{d E^{2}} \hat{\delta}\left(E ; H^{(n)}, H_{0}^{(n)}\right)}{c(n d) E^{n d / 2-2}+\frac{d^{2}}{d E^{2}} \hat{\delta}\left(E ; H^{(n)}, H_{0}^{(n)}\right)}
$$

with $c(n d)=\Gamma(n d / 2-1)^{-1} \pi\left(m / 2 \pi \hbar^{2}\right)^{n d / 2}$. It is a consequence of (3.2) and (3.9). It would be interesting to analyze this expression and, in particular, the quantity $\hat{\delta}\left(E ; H^{(n)}, H_{0}^{(n)}\right)$ when $E$ approaches the bottom of the spectrum of $H^{(n)}$.

Similarly to our discussion of the classical case we want to argue that at least for periodic or random potentials, which in addition are twice differentiable, the heat capacity is positive for all large energies. For this we use the large energy asymptotics of the phase shift [71, 38, 65] and on which we shall comment below. In the present context this asymptotics takes the form (assuming in addition that $V$ is twice differentiable in the periodic case or that the single site potential $V_{0}$ is twice differentiable and has support strictly inside the unit cube in the random case) we have

$$
\frac{1}{|\Lambda|^{n} \pi} \delta\left(E ; H_{0}^{(n)}+V_{\Lambda^{n}}^{(n)}, H_{0}^{(n)}\right)=\frac{1}{|\Lambda|^{n}} \mathfrak{n}\left(E ; H_{0}^{(n)}\right)\left(a_{1}(n) E^{-1}+a_{2}(n) E^{-2}+O\left(E^{-3}\right)\right)
$$

with

$$
\begin{aligned}
a_{1}(n) & =-\frac{n d}{2} \int V_{\Lambda^{n}}^{(n)}(x) d^{n d} x=-n \frac{n d}{2} \frac{1}{|\Lambda|} \int V_{\Lambda}(x) d^{d} x, \\
a_{2}(n) & =\frac{n d}{4}\left(\frac{n d}{2}-1\right) \int V_{\Lambda^{n}}^{(n)}(x)^{2} d^{n d} x \\
& =\frac{n d}{4}\left(\frac{n d}{2}-1\right)\left[\frac{n}{|\Lambda|} \int V_{\Lambda}(x)^{2} d^{d} x+\frac{n(n-1)}{|\Lambda|^{2}}\left(\int V_{\Lambda}(x) d^{d} x\right)^{2}\right] .
\end{aligned}
$$

What has been proved so far is, e.g., that for periodic or random potentials in one dimension there is an upper bound for the phase shift density of the form const $\cdot E^{-1 / 2}$ [47]. In threedimensional case the three-term asymptotics of the integrated density of states is known [40].

By the Schwarz inequality

$$
\frac{1}{|\Lambda|^{2}}\left(\int V_{\Lambda}(x) d^{d} x\right)^{2} \leq \frac{1}{|\Lambda|} \int V_{\Lambda}^{2}(x) d^{d} x
$$

with equality if and only if $V$ is constant on $\Lambda$. Both terms stay bounded when $\Lambda \rightarrow \infty$. Let $\bar{V}^{2}$ and $\overline{V^{2}}$, respectively, denote these limits as $\Lambda \rightarrow \infty$. They agree with the quantities 
given by (2.39). In the random case this limit is deterministic, a trivial example of selfaveraging.

Combined with (3.2) and (3.9), and under the assumption that the asymptotic expansion is preserved in the limit $\Lambda \rightarrow \infty$, relation (3.16) gives the following asymptotic behavior of the integrated density of states

$$
\begin{aligned}
\mathfrak{n}\left(E ; H_{0}^{(n)}+V^{(n)}\right)= & \mathfrak{n}\left(E ; H_{0}^{(n)}\right)\left(1-E^{-1} n \frac{n d}{2} \bar{V}\right. \\
& \left.+\frac{E^{-2}}{2} \frac{n d}{2}\left(\frac{n d}{2}-1\right)\left[n \overline{V^{2}}+n(n-1) \bar{V}^{2}\right]+O\left(E^{-3}\right)\right) .
\end{aligned}
$$

Observe that $\hbar$ does not appear explicitly in this asymptotic expansion. Therefore it makes sense to compare it with the corresponding classical case. Indeed, when we multiply (3.18) by $h^{\text {nd }}$ the resulting derivative w.r.t. $E$ agrees with the derivative of $\mathfrak{n}\left(E ; H_{\mathrm{cl}}^{(n)}\right)$. This follows (3.2) and (2.38) and has the important consequence that to this given order the asymptotic energy-temperature relation (2.41) as well as the asymptotic relation (2.42) for the heat capacity and the conclusions thereof carry over to this quantum case. Moreover, it is natural to conjecture the classical limit relation

$$
\lim _{\hbar \downarrow 0} \mathfrak{n}\left(E ; H^{(n)}\right) h^{\text {nd }}=\mathfrak{n}\left(E ; H_{\mathrm{cl}}^{(n)}\right) .
$$

For relations in this vein when the classical theory describes ergodic motion, see, e.g., [11, 73, 74]. In addition, by the example in the previous section of a classical model with negative specific heat, with an appropriate control of this limit in (3.19) this could lead to a quantum model with negative specific heat for small $\hbar$.

We turn to a discussion of the canonical ensemble in the quantum case. In analogy to the classical case (see (2.15)) the Boltzmann-Gibbs canonical partition function for $H_{\Lambda}^{(n)}$ may also be given in terms of $N\left(E ; H_{\Lambda}^{(n)}\right)$ as

$$
Z\left(\beta ; H_{\Lambda}^{(n)}\right)=\operatorname{tr}_{L^{2}\left(\Lambda^{n}\right)}\left(e^{-\beta H_{\Lambda}^{(n)}}\right)=\int e^{-\beta E} d N\left(E ; H_{\Lambda}^{(n)}\right)=\beta \int e^{-\beta E} N\left(E ; H_{\Lambda}^{(n)}\right) d E
$$

with mean energy

$$
-\frac{d}{d \beta} \ln Z\left(\beta ; H_{\Lambda}^{(n)}\right)=\frac{\int E e^{-\beta E} d N\left(E ; H_{\Lambda}^{(n)}\right)}{Z\left(\beta ; H_{\Lambda}^{(n)}\right)} .
$$

Again by the Schwarz inequality it is a monotone increasing function of the temperature.

So as in the classical case when $\Lambda \rightarrow \infty$ it therefore makes sense to consider the following partition function per unit interaction volume

$$
0 \leq z\left(\beta ; H^{(n)}\right)=\lim _{\Lambda \rightarrow \infty} \frac{1}{|\Lambda|^{n}} Z\left(\beta ; H_{\Lambda}^{(n)}\right)=\int e^{-\beta E} d \mathfrak{n}\left(E ; H^{(n)}\right)=\beta \int e^{-\beta E} \mathfrak{n}\left(E ; H^{(n)}\right) d E .
$$

The mean energy is then given as

$$
\begin{aligned}
\bar{e}\left(\beta ; H^{(n)}\right)=-\frac{d}{d \beta} \ln z\left(\beta ; H^{(n)}\right) & =\frac{\int E e^{-\beta E} d \mathfrak{n}\left(E ; H^{(n)}\right)}{z\left(\beta ; H^{(n)}\right)} \\
& =-\frac{1}{\beta}+\frac{\beta}{z\left(\beta ; H^{(n)}\right)} \int E e^{-\beta E} \mathfrak{n}\left(E ; H^{(n)}\right) d E,
\end{aligned}
$$

which is not necessarily positive since $\mathfrak{n}\left(E ; H^{(n)}\right)$ may be non-zero for $E<0$. Again by the Schwarz inequality the mean energy for the canonical distribution is a monotone increasing 
function of the temperature. For the free case we obtain the familiar relation

$$
z\left(\beta ; H_{0}^{(n)}\right)=\left(\frac{m}{2 \pi \hbar^{2} \beta}\right)^{n d / 2}=\frac{1}{h^{n}}(2 \pi m k T)^{n d / 2}
$$

and hence the energy-temperature relation $\bar{e}\left(\beta ; H_{0}^{(n)}\right)=(n d / 2) k T$ which agrees with the one in the microcanonical ensemble (3.14) when $n$ is large. By (3.9) (compare also with the relation (3.8) ) in the one-particle case, we obtain the relation

$$
z\left(\beta ; H^{(n)}\right)-z\left(\beta ; H_{0}^{(n)}\right)=\frac{1}{\pi} \int e^{-\beta E} d \hat{\delta}\left(E ; H^{(n)}, H_{0}^{(n)}\right)=\frac{\beta}{\pi} \int e^{-\beta E} \hat{\delta}\left(E ; H^{(n)}, H_{0}^{(n)}\right) d E .
$$

The relations (3.23) and 3.24) give

$$
\begin{aligned}
\bar{e}\left(\beta ; H^{(n)}\right)-\bar{e}\left(\beta ; H_{0}^{(n)}\right)=-\frac{d}{d \beta} \ln \frac{z\left(\beta ; H^{(n)}\right)}{z\left(\beta ; H_{0}^{(n)}\right)} \\
=-\frac{d}{d \beta} \ln \left(1+\left(\frac{2 \pi \hbar^{2} \beta}{m}\right)^{n d / 2} \frac{1}{\pi} \int e^{-\beta E} d \hat{\delta}\left(E ; H^{(n)}, H_{0}^{(n)}\right)\right) \\
=-\left(\frac{2 \pi \hbar^{2} \beta}{m}\right)^{n d / 2}\left(1+\left(\frac{2 \pi \hbar^{2} \beta}{m}\right)^{n d / 2} \frac{1}{\pi} \int e^{-\beta E} d \hat{\delta}\left(E ; H^{(n)}, H_{0}^{(n)}\right)\right)^{-1} \\
\quad \cdot \frac{1}{\pi} \int\left(\frac{n d}{2} \beta^{-1}-E\right) e^{-\beta E} d \hat{\delta}\left(E ; H^{(n)}, H_{0}^{(n)}\right) .
\end{aligned}
$$

This relation corresponds to the temperature difference 3.15 ) in the microcanonical description and again the right hand side involves the potential only through the scattering phase density. We do not know at present under which conditions one may take the limit $T \rightarrow 0$ in (3.25) and what the result should be. As in the microcanonical case this presumably requires an analysis of the scattering phase density near the bottom of the spectrum of $H^{(n)}$ which equals $n \min (\inf \operatorname{spec}(H), 0)$ (see the remark after (3.32) below).

We note in this context that a theorem of Fumi [23] (see also [9, 22, 16, 55]) relates the shift of the ground state energy due to a single impurity of a system of non-interacting fermions to the scattering phase caused by this impurity by zero temperature. We may recover Fumi's theorem now in the form of a statement on the shift of the mean energy density for non-interacting electrons, moving in a periodic or random potential $V$, from the general relation (3.7) in the following way. For given $V$ and given electron density $\mathrm{n}$ let the Fermi energy $E_{F}=E_{F}(\mathrm{n} ; H)$ be given as the solution to the equation $\mathrm{n} / 2=\mathfrak{n}\left(E_{F} ; H\right)$, where the factor $1 / 2$ accounts for the spin. Thus $E_{F}\left(\mathrm{n} ; H_{0}\right)=\left(\mathrm{n} h^{d} \Gamma(d / 2+1) / 2\right)^{2 / d} /(2 \pi m)$ for the free theory. Then the ground state energy density $\Omega_{F}$ is given as

$$
\Omega_{F}(\mathrm{n} ; H)=2 \int_{\inf \operatorname{spec}(H)}^{E_{F}(\mathrm{n} ; H)} E d \mathfrak{n}(E ; H)=-2 \int_{\inf \operatorname{spec}(H)}^{E_{F}(\mathrm{n} ; H)} \mathfrak{n}(E ; H) d E+E_{F}(\mathrm{n} ; H) \mathrm{n} .
$$

Here again the factor 2 accounts for the spin and the last relation follows by partial integration. For the free theory we have $\Omega_{F}\left(\mathrm{n} ; H_{0}\right)=d /(2(d / 2+1)) E_{F}\left(\mathrm{n} ; H_{0}\right) \mathrm{n}$.

We recall that $\mathfrak{n}(E ; H)$ is a monotone increasing function in $E$ but for periodic $V$ it is constant outside the spectral bands, so $E_{F}$ may not be uniquely defined if $\mathrm{n}$ is such that $E_{F}(\mathrm{n} ; H)$ lies outside the bands. But by the same observation and the first equality in (3.26) $\Omega_{F}(\mathrm{n} ; H)$ is still unique. For random potentials with no gaps in the spectrum the deterministic quantity $\mathfrak{n}(E ; H)$ is strictly increasing, so there is no problem then with the definition of $E_{F}(\mathrm{n} ; H)$. 
From 3.26 , we obtain the following relation for the shift of the mean energy density w.r.t. the free theory as

$$
\begin{aligned}
\Omega_{F}(\mathrm{n} ; H)-\Omega_{F}\left(\mathrm{n} ; H_{0}\right)= & 2 \int_{\inf \operatorname{spec}(H)}^{E_{F}(\mathrm{n} ; H)} E d \mathfrak{n}(E ; H)-2 \int_{0}^{E_{F}\left(\mathrm{n} ; H_{0}\right)} E d \mathfrak{n}\left(E ; H_{0}\right) \\
= & -2 \int_{\operatorname{infspec}(H)}^{E_{F}(\mathrm{n} ; H)} \mathfrak{n}(E ; H) d E-2 \int_{0}^{E_{F}\left(\mathrm{n} ; H_{0}\right)} \mathfrak{n}\left(E ; H_{0}\right) d E \\
& +\left(E_{F}(\mathrm{n} ; H)-E_{F}\left(\mathrm{n} ; H_{0}\right)\right) \mathrm{n} \\
\cong & -\frac{2}{\pi} \int_{\min (\inf \operatorname{spec}(H), 0)}^{E_{F}\left(\mathrm{n} ; H_{0}\right)} \hat{\delta}\left(E ; H, H_{0}\right) d E
\end{aligned}
$$

in the approximation where $E_{F}(\mathrm{n} ; H) \cong E_{F}\left(\mathrm{n} ; H_{0}\right)$, which should be valid for weak potentials. Again we note that as in the case of the extension of Friedel's theorem the contribution by several impurities is not just the sum of the contributions of the single impurities. The correct analysis of the contributions by random impurities was performed in our work [45, 46].

Returning to our general discussion of the canonical ensemble an entropy may be defined in the following way. With $\rho\left(\beta ; H_{\Lambda}^{(n)}\right)=\exp \left(-\beta H_{\Lambda}^{(n)}\right) / Z\left(\beta ; H_{\Lambda}^{(n)}\right)$ being the density matrix (i.e., $0 \leq \rho\left(\beta ; H_{\Lambda}^{(n)}\right)$ and $\operatorname{tr}_{L^{2}\left(\Lambda^{n}\right)} \rho\left(\beta ; H_{\Lambda}^{(n)}\right)=1$ ) associated to the canonical theory for $H_{\Lambda}^{(n)}$ at temperature $T$, let

$$
\begin{aligned}
0 \leq S\left(\beta ; H_{\Lambda}^{(n)}\right) & =-k \operatorname{tr}_{L^{2}\left(\Lambda^{n}\right)}\left(\rho\left(\beta ; H_{\Lambda}^{(n)}\right) \ln \rho\left(\beta ; H_{\Lambda}^{(n)}\right)\right) \\
& =k \ln Z\left(\beta ; H_{\Lambda}^{(n)}\right)+\frac{k \beta}{Z\left(\beta ; H_{\Lambda}^{(n)}\right)} \int E e^{-\beta E} d N\left(E ; H_{\Lambda}^{(n)}\right) \\
& =-k \int Z\left(\beta ; H_{\Lambda}^{(n)}\right)^{-1} e^{-\beta E} \ln \left(Z\left(\beta ; H_{\Lambda}^{(n)}\right)^{-1} e^{-\beta E}\right) d N\left(E ; H_{\Lambda}^{(n)}\right) \\
& =-k \beta^{2} \frac{d}{d \beta}\left(\frac{1}{\beta} \ln Z\left(\beta ; H_{\Lambda}^{(n)}\right)\right)=k \beta^{2} \frac{d}{d \beta} F\left(\beta ; H_{\Lambda}^{(n)}\right)
\end{aligned}
$$

be the resulting von Neumann entropy [57]. Also

$$
F\left(\beta ; H_{\Lambda}^{(n)}\right)=-\frac{1}{\beta} \ln Z\left(\beta ; H_{\Lambda}^{(n)}\right)
$$

is the Helmholtz free energy. Recall that $S\left(\beta ; H_{\Lambda}^{(n)}\right) \geq 0$ holds because $-x \ln x \geq 0$ for $0 \leq x \leq 1$ and all eigenvalues of $\rho\left(\beta ; H_{\Lambda}^{(n)}\right)$ lie between 0 and 1 . By the second relation in (3.27) the limit

$$
s\left(\beta ; H^{(n)}\right)=\lim _{\Lambda \rightarrow \infty}\left(S\left(\beta ; H_{\Lambda}^{(n)}\right)-k n \ln |\Lambda|\right)
$$

exists and is given as

$$
\begin{aligned}
s\left(\beta ; H^{(n)}\right) & =k \ln z\left(\beta ; H^{(n)}\right)+\frac{k \beta}{z\left(\beta ; H^{(n)}\right)} \int E e^{-\beta E} d \mathfrak{n}\left(E ; H^{(n)}\right) \\
& =-k \int z\left(\beta ; H^{(n)}\right)^{-1} e^{-\beta E} \ln \left(z\left(\beta ; H^{(n)}\right)^{-1} e^{-\beta E}\right) d \mathfrak{n}\left(E ; H^{(n)}\right) \\
& =-k \beta^{2} \frac{d}{d \beta}\left(\frac{1}{\beta} \ln z\left(\beta ; H^{(n)}\right)\right) .
\end{aligned}
$$

We interpret this as the entropy per unit interaction volume. Since $S\left(\beta ; H_{\Lambda}^{(n)}\right)$ is a monotone decreasing function in $\beta$ so is $s\left(\beta ; H^{(n)}\right)$ by (3.28). 
By (3.23) for the free case we have

$$
s\left(\beta ; H_{0}^{(n)}\right)=\frac{n d}{2} k\left(1+\ln \frac{m}{\hbar^{2} \beta}\right)
$$

so in particular $s\left(\beta ; H_{0}^{(n)}\right)$ tends to $+\infty$ as $\beta \rightarrow 0$ and to $-\infty$ as $\beta \rightarrow \infty$. In analogy to the microcanonical case (3.12) the last property is again an undesired feature, which it shares with the classical theory (see, e.g., [76]). The first property continues to hold in the presence of a potential $V$, which in the present context we will assume to be bounded. The way to see this is to consider the difference $s\left(\beta ; H^{(n)}\right)-s\left(\beta ; H_{0}^{(n)}\right)$. It has an asymptotic expansion in $\beta$ for $\beta$ small, which is obtained as follows. Using the so called heat kernel expansion one can show (see, e.g., [25] and the references given there) that the following asymptotic expansion is valid

$$
\begin{aligned}
\frac{1}{|\Lambda|^{n}} Z\left(\beta ; H_{\Lambda}^{(n)}\right)= & \left(\frac{m}{2 \pi \hbar^{2} \beta}\right)^{n d / 2}\left(1-n \beta \frac{1}{|\Lambda|} \int V_{\Lambda}(x) d^{d} x\right. \\
& \left.+\frac{\beta^{2}}{2}\left[n \frac{1}{|\Lambda|} \int V_{\Lambda}^{2}(x) d^{d} x+\frac{n(n-1)}{|\Lambda|^{2}}\left(\int V_{\Lambda}(x) d^{d} x\right)^{2}\right]+O\left(\beta^{3}\right)\right) .
\end{aligned}
$$

Here we have assumed that $\Lambda$ is a finite union of unit cubes. In addition in the periodic case the potential $V$ is assumed to be twice differentiable and in the random case (2.20) the single site potential $V_{0}$ with support in the unit cube centered at the origin is also twice differentiable and vanishes near the boundary. In case $V$ and $V_{0}$ have higher order derivatives the asymptotic expansion (3.30) also extends to higher orders. Note that apart from the pre-factor Planck's constant does not appear. This is a consequence of the assumptions just made on $V, V_{0}$ and on $\Lambda$. In general, powers of $\hbar$ appear combined with derivatives of $V$ and $V_{\omega}$ of the same order within integrals over $\Lambda$. Integration over $\Lambda$ therefore gives boundary contributions which vanish.

Assuming again that this asymptotic expansion is preserved in the limit $\Lambda \rightarrow \infty$, we obtain

$$
z\left(\beta ; H^{(n)}\right)=z\left(\beta ; H_{0}^{(n)}\right)\left(1-n \beta \bar{V}+\frac{\beta^{2}}{2}\left[n \overline{V^{2}}+n(n-1) \bar{V}^{2}\right]+O\left(\beta^{3}\right)\right) .
$$

Again up to a factor $h^{\text {nd }}$ this agrees with $(2.46)$. Using (3.21) we see that $(3.31$ ) is compatible with (3.18), i.e., the Laplace-Stieltjes transform of (3.18) gives (3.31). As a matter of fact, the conjecture made in [71] on the asymptotic behavior of the phase shift (which in the present context is (3.16) ) was just made to fit in this way with the "heat kernel" expansion at large temperatures (small $\beta$ ) of

$$
\frac{1}{|\Lambda|^{n}} \operatorname{tr}_{L^{2}\left(\mathbb{R}^{n d}\right)}\left(e^{-\beta\left(H_{0}^{(n)}+V_{\Lambda^{n}}^{(n)}\right.}-e^{-\beta H_{0}^{(n)}}\right) .
$$

For comparison note that in general no asymptotic expansion beyond the famous Weyl term for the number of eigenvalues of an elliptic differential operator on a compact manifold like $N\left(E ; H_{\Lambda}^{(n)}\right)$ is known. The best result in this direction is due to Hörmander [34]. Although related, the conjecture (3.16) is of a different nature since instead of the operator $H_{\Lambda}^{(n)}$ it involves the operator $H_{0}^{(n)}+V_{\Lambda^{n}}^{(n)}$.

Observe that apart from the overall factor so far $\hbar$ does not appear in the asymptotic expansion of $z\left(\beta ; H^{(n)}\right)$ to this order. This is of course in agreement with our corresponding result in the microcanonical setup. Although we shall not need it, we remark that for finite range, smooth potentials $W$ in $d$ dimensions the trace of the heat kernel $\exp \left\{-\beta\left(H_{0}+\right.\right.$ 
$W)\}$ multiplied by $h^{d}$ and for fixed $\beta$ has a well known asymptotic expansion in (positive) powers of $h$ (see, e.g., [50]) which has been shown to be a complete asymptotic expansion in [31, 72]. For the asymptotics of the integrated density of states for periodic Schrödinger operators we refer to [40].

Relation (3.31) results in the following relation for the entropy

$$
s\left(\beta ; H^{(n)}\right)-s\left(\beta ; H_{0}^{(n)}\right)=-k \beta^{2} \frac{d}{d \beta} \frac{1}{\beta} \ln \frac{z\left(\beta ; H^{(n)}\right)}{z\left(\beta ; H_{0}^{(n)}\right)}=-k n \frac{\beta^{2}}{2}\left(\overline{V^{2}}-\bar{V}^{2}\right)+O\left(\beta^{3}\right) .
$$

By (3.17) the first term on the right hand side of (3.32) is strictly negative if $V \neq 0$. We do not now about a similar asymptotic expansion in $1 / \beta$, which is uniform in $\Lambda$. The problem arises because as mentioned above it is difficult to establish the asymptotic behavior of (3.25) for small $T$. Note that for fixed $\Lambda$ one has, e.g.,

$$
\ln Z\left(\beta ; H_{\Lambda}^{(n)}\right)=-\beta \varepsilon_{0}+\ln \operatorname{dim} P_{0}+\frac{\operatorname{dim} P_{1}}{\operatorname{dim} P_{0}} e^{-\beta\left(\varepsilon_{1}-\varepsilon_{0}\right)}+O\left(e^{-\beta\left(\varepsilon_{2}-\varepsilon_{1}\right)}\right),
$$

where $\varepsilon_{0}<\varepsilon_{1}<\varepsilon_{2}<\ldots$ are the different eigenvalues of $H_{\Lambda}^{(n)}$ and $P_{i}$ the orthogonal projections onto the corresponding eigenspaces such that $\operatorname{dim} P_{i}$ are the multiplicities of the eigenvalues. For fixed $i$ the difference $\varepsilon_{i}-\varepsilon_{0}$ tends to zero at least as $|\Lambda|^{1 / d}$ as $\Lambda \rightarrow \infty$.

Define

$$
f\left(\beta ; H^{(n)}\right)=-\frac{1}{\beta} \lim _{\Lambda \rightarrow \infty}\left(F\left(\beta ; H_{\Lambda}^{(n)}\right)-n|\Lambda|\right)=-\frac{1}{\beta} \ln z\left(\beta ; H^{(n)}\right)
$$

to be the Helmholtz free energy per interaction volume. From (3.22) and 3.29 ) we obtain the relation between the mean energy, the entropy and the free energy, familiar in the usual context of the canonical ensemble (compare with the same relation (2.43) in the classical case)

$$
\bar{e}\left(\beta ; H^{(n)}\right)=T s\left(\beta ; H^{(n)}\right)+f\left(\beta ; H^{(n)}\right) .
$$

By (3.30) we have

$$
f\left(\beta ; H^{(n)}\right)-f\left(\beta ; H_{0}^{(n)}\right)=n \bar{V}-n \frac{\beta}{2}\left[\overline{V^{2}}-\frac{1}{2} \bar{V}^{2}\right]+O\left(\beta^{2}\right)
$$

for small $\beta$ and where $f\left(\beta ; H_{0}^{(n)}\right)$ may be read off $(3.23)$. The mean energy has the asymptotic form

$$
\bar{e}\left(\beta ; H^{(n)}\right)=\frac{n d}{2} \beta^{-1}+n \bar{V}-\frac{n \beta}{2}\left[\overline{V^{2}}-\bar{V}^{2}\right]+O\left(\beta^{2}\right)
$$

which agrees with the asymptotic behavior (2.47) in the classical canonical description. Therefore the inverse asymptotic relation (2.48) carries over and so for large $E$ and $n$ these microcanonical and canonical descriptions are approximately equal. In other words in analogy to 2.49) we have the approximative relations which are inverse to each other

$$
T \approx T\left(\bar{e}\left(\beta=1 / k T ; H^{(n)}\right)\right), \quad E \approx \bar{e}\left(\beta=1 / k T\left(E ; H^{(n)}\right) ; H^{(n)}\right) .
$$

Also in this canonical setup the heat capacity is defined as

$$
c_{\mathrm{v}}\left(\beta ; H^{(n)}\right)=\frac{d}{d T} \bar{e}\left(\beta ; H^{(n)}\right)=\frac{1}{k T^{2}} \Delta \bar{e}\left(\beta ; H^{(n)}\right)^{2},
$$

which is $\geq 0$ by the discussion above and which equals $k n d / 2$ for all $\beta$ in the free case as it should. As in (2.50)

$$
\Delta \bar{e}\left(\beta ; H^{(n)}\right)=\left(\frac{d^{2}}{d \beta^{2}} \ln z\left(\beta ; H^{(n)}\right)\right)^{1 / 2}
$$


is the energy fluctuation. So for small $\beta$ we obtain

$$
c_{\mathrm{v}}\left(\beta ; H^{(n)}\right)=\frac{k n d}{2}+\frac{k n \beta^{2}}{2}\left[\overline{V^{2}}-\bar{V}^{2}\right]+O\left(\beta^{3}\right)
$$

and thus for small $\beta$ the heat capacity increases when an external potential $V$ is switched on. By (3.34) relation (3.36) in turn gives the following asymptotic behavior for the energy fluctuation

$$
\Delta \bar{e}\left(\beta ; H^{(n)}\right)=\sqrt{\frac{n d}{2}} \frac{1}{\beta}\left(1+\frac{\beta^{2}}{d}\left[\overline{V^{2}}-\bar{V}^{2}\right]+O\left(\beta^{3}\right)\right),
$$

which by (3.35) may also be obtained from (3.31) and which agrees with its classical counterpart (2.51).

In analogy to our analysis in the classical case (see the discussion following (2.44)) and by the same arguments (involving the assumption that the heat capacity is sufficiently large and positive), also in this quantum context the canonical and the microcanonical ensemble both give the same energy-temperature relation and the same heat capacities. In particular the variance $\alpha$ of the approximating Gauss distribution is just the square of the energy fluctuation (3.35), which in turn is related to the heat capacity by (3.34). Indeed, by observing the necessary differentiability conditions the discussion can be carried over verbatim.

We now want to establish that for given one particle theory with a periodic external potential smoothness of $\mathfrak{n}\left(E ; H^{(n)}\right)$ increases with the particle number $n$ and that the derivatives are all nonnegative. For this the next relation will become relevant. Observe first that for all $n \geq 2,1 \leq k \leq n-1, \Lambda$ and $E$

$$
N\left(E ; H_{\Lambda}^{(n)}\right)=\int N\left(E-E^{\prime} ; H_{\Lambda}^{(k)}\right) d N\left(E^{\prime} ; H_{\Lambda}^{(n-k)}\right) .
$$

This follows easily from the identity

$$
\Theta\left(E-\varepsilon-\varepsilon^{\prime}\right)=\int \Theta\left(E-E^{\prime}-\varepsilon\right) \delta\left(E^{\prime}-\varepsilon^{\prime}\right) d E^{\prime}=\int \Theta\left(E-E^{\prime}-\varepsilon\right) d \Theta\left(E^{\prime}-\varepsilon^{\prime}\right)
$$

and from $H_{\Lambda}^{(n)}=H_{\Lambda}^{(k)} \otimes \mathbb{I}_{L^{2}\left(\Lambda^{n-k}\right)}+\mathbb{I}_{L^{2}\left(\Lambda^{k}\right)} \otimes H_{\Lambda}^{(n-k)}$ and $\operatorname{tr}_{L^{2}\left(\Lambda^{n}\right)}(A \otimes B)=\operatorname{tr}_{L^{2}\left(\Lambda^{k}\right)}(A) \operatorname{tr}_{L^{2}\left(\Lambda^{n-k}\right)}(B)$. Therefore the integrated density of states for the $n$-particle theory obeys a similar relation, i.e., it may be written as a Riemann-Stieltjes convolution

$$
\mathfrak{n}\left(E ; H^{(n)}\right)=\int \mathfrak{n}\left(E-E^{\prime} ; H^{(k)}\right) d \mathfrak{n}\left(E^{\prime} ; H^{(n-k)}\right)=\mathfrak{n}\left(\cdot ; H^{(k)}\right) *_{S} \mathfrak{n}\left(\cdot ; H^{(n-k)}\right)(E) .
$$

In particular $\mathfrak{n}\left(E ; H^{(n)}\right)$ is an $(n-1)$-fold iterated Riemann-Stieltjes convolution of the oneparticle integrated density of states $\mathfrak{n}\left(E ; H^{(n=1)}\right)$ with itself. Here we used the assumption that $V \geq-c$ for some $c \geq 0$, such that $H^{(n)} \geq-n c$ and hence $\mathfrak{n}\left(E, H^{(n)}\right)=0$ for all $E<-n c$. Let $E_{\text {thresh }}^{(n)}>-c n$ be the infimum of all $E$ such that $\mathfrak{n}\left(E ; H^{(n)}\right) \neq 0$. Since $\mathfrak{n}(E ; H)$ is Hölder continuous and nonnegative, the Riemann-Stieltjes convolution of $\mathfrak{n}(E ; H)$ with itself exists and is also Hölder continuous. This is easily established with techniques used in, e.g., [24, 17]. Relation (3.37) implies

$$
z\left(\beta ; H^{(n)}\right)=z\left(\beta ; H^{(k)}\right) z\left(\beta ; H^{(n-k)}\right)
$$

giving in particular $z\left(\beta ; H^{(n)}\right)=z(\beta ; H)^{n}$ and the obvious relation $f\left(\beta ; H^{(n)}\right)=n f(\beta ; H)$ for the free energy. Relations of this type are of course familiar in the ordinary quantum canonical ensemble formulation of noninteracting particles. Thus we have, e.g., for the partition function given by 3.20 )

$$
Z\left(\beta ; H_{\Lambda}^{(n)}\right)=Z\left(\beta ; H_{\Lambda}^{(n-k)}\right) Z\left(\beta ; H_{\Lambda}^{(k)}\right) .
$$


With reasonable assumptions on $\mathfrak{n}(E ; H)$ concerning the van Hove singularities when $V$ is a periodic potential and which hold in $d=1$ dimension and for the Kronig- Penney model (see, e.g., [1], for a survey of spectral properties for periodic potentials in general see [39]) we will show in the appendix that $\mathfrak{n}\left(E ; H^{(n=2)}\right)$ is actually continuously differentiable except for a discrete set of energies (without finite accumulation points) where, however, the right and left derivatives exist. As a consequence $\mathfrak{n}\left(E ; H^{(n=3)}\right)$ is continuously differentiable at all energies. Using (3.37) by complete induction we obtain that $\mathfrak{n}\left(E ; H^{(n)}\right)$ is $[(n-1) / 2]$ times continuously differentiable in $E$ with the representation for the $l$ 'th derivative $(l \leq$ $[(n-1) / 2])$ as

$$
\begin{aligned}
\mathfrak{n}^{(l)}\left(E ; H^{(n)}\right) & =\int \mathfrak{n}^{(l-1)}\left(E-E^{\prime} ; H^{(n-2)}\right) d \mathfrak{n}^{\prime}\left(E^{\prime} ; H^{(2)}\right) \\
& =\mathfrak{n}^{(l-1)}\left(\cdot ; H^{(n-2)}\right) *_{S} \mathfrak{n}^{\prime}\left(\cdot ; H^{(2)}\right)(E) .
\end{aligned}
$$

Also from (3.38) it follows again by complete induction in $l$ that these derivatives are all nonnegative. In particular $\mathfrak{n}\left(E ; H^{(n)}\right)$ is concave in $E$ for $n \geq 5$. This increase of smoothness with $n$ is related to the known increase of smoothness with the dimension $d$ of space. Indeed write $H_{0}^{d}$ to denote the dependence of the free Hamiltonian on the space dimension. Then for given external one particle potential $V$ we have $\left(H_{0}^{d}+V\right)^{(n)}=$ $H_{0}^{n d}+V^{(n)}$, where as in the classical case the potential $V^{(n)}$ is given as $V^{(n)}(x)=\sum_{i} V\left(x_{i}\right)$ for $x=\left(x_{1}, x_{2}, \ldots, x_{n}\right) \in \mathbb{R}^{n d}$ and with $x_{i} \in \mathbb{R}^{d}$. Note that periodicity extends in the sense that e.g. $V^{(n)}(x+j)=V^{(n)}(x)$ holds for any $j \in \mathbb{Z}^{n d}$ and $x \in \mathbb{R}^{n d}$, whenever $V(y+i)=V(y)$ for all $i \in \mathbb{Z}^{d}$ and $y \in \mathbb{R}^{d}$.

To sum up by our preceding discussion for one particle Schrödinger operators with periodic potentials we therefore may define a temperature for the microcanonical ensemble for all $H^{(n)}, n \geq 5$. Since the third derivative of $\mathfrak{n}\left(E ; H^{(n)}\right), n \geq 7$ is non-negative it can not vanish identically on any interval $\left[E_{\text {thresh }}^{(n)}, E\right]$. To see this, assume the contrary. Then by integrating 3 times we see that $\mathfrak{n}\left(E ; H^{(n)}\right)$ also would vanish identically on this interval, which is not possible. So again by integrating, the first and second derivatives of $\mathfrak{n}\left(E ; H^{(n)}\right), n \geq 5$ are strictly positive for any $E>E_{\text {thresh. }}^{(n)}$. So we conclude that the microcanonical temperature for $H^{(n)}, n \geq 5$ is actually positive, finite and a continuous function of $E$. Similarly, since the fourth derivative of the density of states for $H^{(n)}, n \geq 9$ is non-negative, the second derivative is concave in $E$. However, this is not sufficient to conclude that $1 / T$ is a concave function of $E$ whenever $n \geq 9$ and presently we do not know sufficient conditions on the potential $V$ which ensure this.

We expect similar results to be valid for the case of stochastic potentials, where it is known that the integrated density of states is Lipschitz continuous, i.e., Hölder continuous of index 1 (see [12]), so one has smoother properties than in the periodic case. For easier, recent proofs that they are Hölder continuous of any index smaller than 1, see [13, 48]. Unfortunately, in general the Riemann-Stieltjes convolution does not improve Hölder continuity or any other similar kind of regularity (see, e.g., [24, 17]). It would be interesting to find additional properties of the integrated density of states which allows one to conclude that their Riemann-Stieltjes convolution improves regularity.

So far we have not taken Bose-Einstein or Fermi-Dirac statistics into account. To do this observe first that the permutation group $S_{n}$ of $n$ elements has a canonical unitary representation $\pi \rightarrow U(\pi), \pi \in S_{n}$ on $\otimes_{n} L^{2}\left(\mathbb{R}^{d}\right)$. A similar observation is valid when $\mathbb{R}^{d}$ is replaced by $\Lambda$. The orthogonal projections onto the subspaces of symmetric (+) and 
antisymmetric (-) wave-functions $\otimes_{n, \pm} L^{2}\left(\mathbb{R}^{d}\right)$ are given as

$$
P_{ \pm}^{(n)}=\frac{1}{n !} \sum_{\pi \in S_{n}}(\operatorname{sign} \pi)^{(1 \mp 1) / 2} U(\pi)
$$

respectively, i.e., $\otimes_{n, \pm} L^{2}\left(\mathbb{R}^{d}\right)=P_{ \pm}^{(n)} \otimes_{n} L^{2}\left(\mathbb{R}^{d}\right)$. This gives $\operatorname{tr}_{\otimes_{n \pm \pm} L^{2}\left(\mathbb{R}^{d}\right)}(A)=\operatorname{tr}_{\otimes_{n} L^{2}\left(\mathbb{R}^{d}\right)}\left(P_{ \pm}^{(n)} A\right)$ and similarly with $\mathbb{R}^{d}$ being replaced by $\Lambda$. Then one may show that the resulting density of states are given by

$$
n_{ \pm}\left(E ; H^{(n)}\right)=\lim _{\Lambda \rightarrow \infty} \frac{1}{|\Lambda|^{n}} \operatorname{tr}_{L^{2}\left(\Lambda^{n}\right)}\left(P_{ \pm}^{(n)} \Theta\left(E-H_{\Lambda}^{(n)}\right)\right)=\frac{1}{n !} \mathfrak{n}\left(E ; H^{(n)}\right),
$$

i.e., when inserting (3.39) into the middle term in $(3.40)$ the contributions from $\pi \neq i d$ vanish in the limit. In the periodic case and in analogy to (3.6) one also has

$$
n_{ \pm}\left(E ; H^{(n)}\right)=\operatorname{tr}_{L^{2}\left(\mathbb{R}^{n d}\right)}\left(\chi_{\Lambda_{0}^{n}} P_{ \pm}^{(n)} \Theta\left(E-H^{(n)}\right)\right) .
$$

In particular the resulting temperature in the microcanonical ensemble does not depend on the statistics. Similarly the partition function in the canonical ensemble is modified by a factor $1 / n !$.

To conclude this section we briefly describe the associated grand canonical ensemble for both statistics using the integrated density of states. In a finite volume $\Lambda$ the partition function for the grand canonical ensemble is given as

$$
Z_{ \pm}(\beta, \mu ; H)=1+\sum_{n=1}^{\infty} e^{-\mu \beta n} \operatorname{tr}_{L^{2}\left(\Lambda^{n}\right)}\left(P_{ \pm}^{(n)} e^{-\beta H^{(n)}}\right) .
$$

with $\mu$ being the chemical potential. Standard arguments therefore give

$$
\ln Z_{ \pm}(\beta, \mu ; H)=\operatorname{tr}_{L^{2}\left(\Lambda^{n}\right)}\left(1 \mp e^{-\beta\left(\mu+H_{\Lambda}^{(n=1)}\right)}\right)^{\mp 1}=\int\left(1 \mp e^{-\beta(\mu+E)}\right)^{\mp 1} d N\left(E ; H_{\Lambda}^{(n=1)}\right) .
$$

Hence $z_{ \pm}(\beta, \mu ; H)$ may be defined in terms of the one-particle integrated density of states by

$$
\ln z_{ \pm}(\beta, \mu ; H)=\lim _{\Lambda \rightarrow \infty} \frac{1}{|\Lambda|} \ln Z_{ \pm}(\beta, \mu ; H)=\int\left(1 \mp e^{-\beta(\mu+E}\right)^{\mp 1} d \mathfrak{n}\left(E ; H^{(n=1)}\right) .
$$

The usual relations for the mean energy and the mean particle number may now be obtained easily.

\section{Appendix A.}

In the the first part of the appendix we will establish increasing regularity for the quantum integrated density of states with increasing particle number in the case of a periodic potential. In the second and last part we will show in an example how randomness may serve to increase the smoothness of the classical integrated density of states already in the one particle case.

For periodic potentials $V$, where bands appear, it is well known that the one-particle integrated density of states $\mathfrak{n}\left(E ; H^{(n=1)}\right)$ is constant for $E$ inside the gaps, smooth inside the bands and behaves like $\left|E-E_{i, \pm}\right|^{1 / 2}$ at an upper $E_{i,+}$ or lower edge $E_{i,-}$ of a band, so in particular it is Hölder continuous of index $1 / 2$ everywhere. More precisely we make the following assumption, which has been proven rigorously in $d=1$ dimension, see [53]. Write the bands (finitely or infinitely many) as the closed, pairwise disjoint intervals $\left[E_{i,-}, E_{i,+}\right]$. 
There are smooth functions $n_{i, \pm}(E ; H), E \geq 0$ small, with $n_{i, \pm}(E=0 ; H)=0$ such that for $E$ near $E_{i, \pm}$

$$
\mathfrak{n}(E ; H)=\left\{\begin{array}{ccc}
\left|E-E_{i, \pm}\right|^{1 / 2} n_{i, \pm}\left(\mp\left(E-E_{i, \pm}\right) ; H\right)+c_{i, \pm} & \text { if } & \mp\left(E-E_{i, \pm}\right) \geq 0 \\
c_{i, \pm} & \text { if } & \pm\left(E-E_{i, \pm}\right) \geq 0
\end{array},\right.
$$

where $c_{i, \pm}=\mathfrak{n}\left(E_{i, \pm} ; H\right)$. In particular $\mathfrak{n}(E ; H)$ is Hölder continuous of index $1 / 2$. Also away from the ends $E_{i, \pm}$ of the bands the function $\mathfrak{n}(E ; H)$ is supposed to be smooth. We note that for $d>1$ the Bethe-Sommerfeld conjecture is actually true: As established by Skriganov and Karpeshina, there are only finitely many gaps (see, e.g., [49] for an extensive list of references and [39]).

With these assumptions we will show that $\mathfrak{n}\left(E ; H^{(n=2)}\right)$ is continuously differentiable in $E$ from the right and from the left. Also the right and left derivatives agree except for the discrete set of energies of the form $E=E_{i, \pm}+E_{j, \pm}$. In other words the derivative of $\mathfrak{n}\left(E ; H^{(n=2)}\right)$ is possibly discontinuous at these energies. Furthermore we will show that $\mathfrak{n}\left(E ; H^{(n=3)}\right)$ is differentiable in $E$ for all $E$ with a derivative which is Hölder continuous of index $1 / 2$.

For the proof let $0 \leq \chi_{i, \pm}(E) \leq 1$ be smooth functions which are equal to one near $E_{i, \pm}$ and where A.1 holds. They may be chosen to have non-overlapping support, i.e., any product of two different $\chi_{i, \pm}(E)$ 's is zero. Set $0 \leq \chi(E)=\sum_{i, \pm} \chi_{i, \pm}(E) \leq 1$ and write $\mathfrak{n}_{1}(E ; H)=(1-\chi(E)) \mathfrak{n}(E ; H), \mathfrak{n}_{2}(E ; H)=\chi(E) \mathfrak{n}(E ; H)$ such that $\mathfrak{n}(E ; H)=\mathfrak{n}_{1}(E ; H)+\mathfrak{n}_{2}(E ; H)$. Then $\mathfrak{n}_{1}(E ; H)$ is smooth everywhere and $\mathfrak{n}_{2}(E ; H)$ vanishes outside small intervals around the points $E_{i, \pm}$. With this decomposition of $\mathfrak{n}(E ; H)$ we obtain

(A.2)

$$
\mathfrak{n}\left(E ; H^{2}\right)=\mathfrak{n}_{1}(\cdot ; H) *_{S} \mathfrak{n}_{1}(\cdot ; H)(E)+2 \mathfrak{n}_{1}(\cdot ; H) *_{S} \mathfrak{n}_{2}(\cdot ; H)(E)+\mathfrak{n}_{2}(\cdot ; H) *_{S} \mathfrak{n}_{2}(\cdot ; H)(E) .
$$

The first two terms in (A.2) are easily seen to be continuously differentiable w.r.t. $E$, so we only have to show that the third term is continuously differentiable. It suffices to consider any term of the form

$$
\int \chi_{i, \pm}\left(E-E^{\prime}\right) \mathfrak{n}\left(E-E^{\prime} ; H\right) d\left(\chi_{j, \pm}\left(E^{\prime}\right) \mathfrak{n}\left(E^{\prime} ; H\right)\right),
$$

since for given $E$ there are only finitely many $E_{i, \pm}$ 's with $E_{i, \pm} \leq E$. Now we use the assumption (A.1) and obtain

$$
\begin{aligned}
d\left(\chi_{l, \pm}(E) \mathfrak{n}(E ; H)\right)= & \Theta\left(\mp\left(E-E_{l, \pm}\right)\right)\left(\left|E-E_{l, \pm}\right|^{1 / 2} f_{l, \pm, 1}(E)+\left|E-E_{l, \pm}\right|^{-1 / 2} f_{l, \pm, 2}(E)\right) d E \\
& +f_{l, \pm, 3}(E) d E
\end{aligned}
$$

$l=i$ or $j$, where the $f_{l, \pm, k}$ 's, $k=1,2,3$ are smooth with support contained in the support of $\chi_{l, \pm}$. We insert this into the formal derivative of $(A .3)$ and obtain 9 terms all of which should be finite integrals. The potentially most dangerous one and on which we shall concentrate is

(A.4)

$\int \Theta\left(\mp\left(E-E^{\prime}-E_{i, \pm}\right)\right) \Theta\left(\mp\left(E^{\prime}-E_{j, \pm}\right)\right)\left|E-E^{\prime}-E_{i, \pm}\right|^{-1 / 2}\left|E^{\prime}-E_{j, \pm}\right|^{-1 / 2} f_{i, \pm, 2}\left(E-E^{\prime}\right) f_{j, \pm, 2}\left(E^{\prime}\right) d E^{\prime}$.

For given $i, \pm$ and $j, \pm$ this integral is finite and continuous in $E$ when $E$ stays away from $E_{i, \pm}+E_{j, \pm}$, so let $E=E_{i, \pm}+E_{j, \pm}+\varepsilon$ with $\varepsilon$ small. Now we make the variable transformation $E^{\prime}=E_{j, \pm}+\varepsilon^{\prime}$. Then (A.4) may be written as

$$
\int \Theta\left(\mp\left(\varepsilon-\varepsilon^{\prime}\right)\right) \Theta\left(\mp \varepsilon^{\prime}\right)\left|\varepsilon-\varepsilon^{\prime}\right|^{-1 / 2}\left|\varepsilon^{\prime}\right|^{-1 / 2} g_{1}\left(\varepsilon-\varepsilon^{\prime}\right) g_{2}\left(\varepsilon^{\prime}\right) d \varepsilon^{\prime},
$$


where $g_{1}\left(\varepsilon-\varepsilon^{\prime}\right)=f_{i, \pm, 2}\left(\varepsilon-\varepsilon^{\prime}+E_{i, \pm}\right)$ and $g_{2}\left(\varepsilon^{\prime}\right)=f_{j, \pm, 2}\left(\varepsilon^{\prime}+E_{j, \pm}\right)$ are smooth and vanish outside a neighborhood of the origin. Also the choice of the sign in $\mp$ in the first Heaviside step function refers to $i, \pm$ while the choice of the sign in $\mp$ in the second refers to $j, \pm$. For $\varepsilon \neq 0$ (A.5) is continuous in $\varepsilon$. Also both limits $\varepsilon \downarrow 0$ and $\varepsilon \uparrow 0$ exist but are in general different. In fact, consider the case,++ of the signs, i.e., the choice $i,-$ and $j,-$. Then (A.5) vanishes for $\varepsilon<0$ and for $\varepsilon>0$ we make the variable transformation $\varepsilon^{\prime}=\varepsilon x$ such that A.5 takes the form

$$
\int_{0}^{1}(1-x)^{-1 / 2} x^{-1 / 2} g_{1}(\varepsilon(1-x)) g_{2}(\varepsilon x) d x
$$

which is continuous in $\varepsilon>0$ and has a finite limit equal to

$$
g_{1}(0) g_{2}(0) \int_{0}^{1}(1-x)^{-1 / 2} x^{-1 / 2} d x
$$

when $\varepsilon \downarrow 0$. The other three cases are discussed similarly. This concludes the proof of our claim on the differentiability of $\mathfrak{n}\left(E ; H^{(n=2)}\right)$. To see that $\mathfrak{n}\left(E ; H^{(n=3)}\right)$ has a derivative which is Hölder continuous of index $1 / 2$, we use the representation $(3.38)$ with $n=3$ and $l=1$ for the derivative of $\mathfrak{n}\left(E ; H^{(n=3)}\right)$. Since we just established that $\mathfrak{n}^{\prime}\left(E ; H^{(n=2)}\right)$ is continuous in $E$ except for a discrete set of discontinuities (without finite accumulation points) some easy arguments establish this last claim.

\section{References}

[1] S. Albeverio, F. Gesztesy, R. Høegh-Krohn, and H. Holden, Solvable Models in Quantum Mechanics, Springer, Berlin, 1998.

[2] V.A. Antonov, Most probable phase distribution in spherical stellar systems and conditions on its existence, Vestnik Leningrad State University, Mathematics and Astronomy Series 7 (1962), 135 - 146. [in Russian]

[3] V.A. Antonov, Most probable phase distribution in spherical stellar systems and conditions of its existence, in Dynamics of Star Clusters (J. Goodman and P. Hut, Eds.), D. Reidel, Dordrecht, 1985.

[4] N.W. Ashcroft and N.D. Mermin, Solid State Physics, Saunders College, Philadelphia, 1976.

[5] V.M. Bannur, Dynamical temperatures for quartic and Henon-Heiles oscillators, Phys. Rev. E 58 (1998), $407-409$.

[6] M. D’Agostino, F. Gulminelli, Ph. Chomaz, M. Bruno, F. Canata, R. Bougault, F. Gramegna, I. Iori, N. Le Neindre, G.V. Margagliotti, A. Moroni, and G. Vannini, Negative heat capacity in the critical region of nuclear fragmentation: an experimental evidence of the liquid-gas phase transition, Phys. Lett. B 474 (2000), 219 - 225.

[7] E. Beth and G.E. Uhlenbeck, Quantum theory of the non-ideal gas, Part II, Behavior at low temperatures, Physica, 4 (1937), 914 - 924.

[8] M.Sh. Birman and D.R. Yafaev, The spectral shift function. Work by M.G. Krein and its further development, St. Petersburg Math. J. 4 (1993), $833-870$.

[9] K.A. Brueckner, C.A. Levinson, and H.M. Mahmoud, Two-body forces and nuclear saturation I., Central forces, Phys. Rev. 95 (1954), 217 - 228.

[10] R. Carmona and J. Lacroix, Spectral Theory of random Schrödinger operators, Birkhäuser, Boston, 1990.

[11] Y. Colin de Verdiere, Ergodicite et fonctions propres du laplacien, Commun. Math. Phys. 102 (1985), 497 -502 .

[12] J.-M. Combes and P. Hislop, Localization for some continuous, random Hamiltonians in d dimensions, J. Funct. Anal. 124 (1994), $149-180$.

[13] J.-M. Combes, P. Hislop, and S. Nakamura, The $L^{p}$-theory of the spectral shift function, the Wegner estimate and the integrated density of states for some random operators, Commun. Math. Phys. 218 (2001), 113 130.

[14] R. Courant and D. Hilbert, Methods of Mathematical Physics, Vol. I, Interscience, New York, 1962.

[15] J. Derezinski and C. Gérard, Scattering Theory of Classical and Quantum N-particle systems, Springer, Berlin, 1997.

[16] B.S. deWitt, Transition from discrete to continuous spectra, Phys. Rev. 103 (1956), 1565 - 1571.

[17] R.M. Dudley and R. Norvaiša, Differentiability Of Six Operators on Nonsmooth Functions and p-Variation, Lecture Notes in Mathematics 1703, Springer, Berlin, 1999. 
[18] J.S. Faulkner, Scattering theory and cluster calculations, J. Phys. C: Solid State Phys. 10 (1977), 4661 4670.

[19] M. Fierz, Der Ergodensatz in der Quantenmechanik, Helv. Phys. Acta 28 (1955), 705 - 715.

[20] J. Friedel, The distribution of electron round impurities in monovalent metals, Phil. Mag. 43 (1952), 153 189.

[21] J. Friedel, Electronic structure of primary solid solutions in metals, Adv. Phys. 3 (1953), 446 - 507.

[22] N. Fukuda and R.G. Newton, Energy level shifts in a large enclosure, Phys. Rev. 103 (1956), 1558 - 1564.

[23] F.G. Fumi, Vacancies in monovalent metals, Phil. Mag. 46 (1955), 1007 - 1020.

[24] F.W. Gehring, A study of $\alpha$-variation. I, Trans. Amer. Math. Soc. 76 (1954), 420 - 443.

[25] P.B. Gilkey, Invariance Theory, the Heat Equation and the Atiyah - Singer Index Theorem, Publish or Perish, Wilmington, 1984.

[26] G.M. Graf and D. Schenker, Classical action and quantum N-body asymptotic completeness, in: "Multiparticle Quantum Scattering with Applications to Nuclear, Atomic and Molecular Physics, IMA Volumes in Mathematics and Its Applications", Springer, Berlin, 1997.

[27] S.A. Gredeskul, I.M. Lifschitz, and L. Pastur, Introduction to the theory of disordered systems, John Wiley, New York, 1988.

[28] D.H.E. Gross, Microcanonical thermodynamics and statistical fragmentation of dissipative systems, the topological structure of N-body phase space, Phys. Rep. 279 (1997), 119 - 201.

[29] D.H.E. Gross, Microcanonical Thermodynamics, World Scientific, Singapore, 2001.

[30] D. ter Haar, Statistical mechanics of stellar systems, in Proc. Int. Conf. on Statistical Mechanics, Kyoto (1968), Supplement to J. Phys. Soc. Japan 26 (1969), 25 - 28.

[31] B. Helffer and D. Robert, Calcul fonctionel par la transformée de Mellin et operateurs admissibles, J. Funct. Anal. 53 (1983), 146 - 268.

[32] P. Hertel, H. Narnhofer, and W. Thirring, Thermodynamic functions for fermions with gravostatic and electrostatic interactions, Commun. Math. Phys. 28 (1972), 159 - 176.

[33] P.D. Hislop and F. Klopp, The integrated density of states for some random operators with nonsign definite potentials, preprint (2001).

[34] L. Hörmander, The spectral function of an elliptic operator, Acta Math. 121 (1968), 193 - 218.

[35] K. Huang, Statistical Mechanics, Wiley, New York, 1963.

[36] W. Hunziker and I.M. Sigal, The quantum N-body problem, J. Math. Phys. 41 (2000), 3448 - 3510.

[37] I. Ispolatov and E.G.D. Cohen, On first-order phase transition in microcanonical and canonical nonextensive systems, available from http://arxiv.org/abs/cond-mat/0101311.

[38] A. Jensen, High energy asymptotics for the total scattering phase in potential scattering theory, in: Functional-Analytic Methods for Partial Differential Equations (H. Fujita, T. Ikebe, S.T. Kuroda, Eds.), Lecture Notes in Physics 1450, Springer, Berlin, 1990, p. 187 - 195.

[39] Yu.E. Karpeshina, Perturbation Theory for the Schrödinger Operator with a Periodic Potential, Lecture Notes in Mathematics 1663, Springer, Berlin, 1997.

[40] Yu.E. Karpeshina, On the density of states for the periodic Schrödinger operator, Ark. Mat. 38 (2000), 111 $-137$.

[41] W. Kirsch, Random Schrödinger operators: a course, in: Schrödinger Operators (H. Holden and A. Jensen, Eds.), Lecture Notes in Physics 345, Springer, Berlin, 1989, p. $264-370$.

[42] A.I. Khinchin, Mathematical Foundations of Statistical Mechanics, Dover, New York, 1949.

[43] V. Kostrykin and R. Schrader, Cluster properties of one particle Schrödinger operators, Rev. Math. Phys. 6 (1994), $833-853$.

[44] V. Kostrykin and R. Schrader, Cluster properties of one particle Schrödinger operators. II, Rev. Math. Phys. 10 (1998), $627-683$.

[45] V. Kostrykin and R. Schrader, Scattering theory approach to random Schrödinger operators in one dimension, Rev. Math. Phys. 11 (1999), 187 - 242.

[46] V. Kostrykin and R. Schrader, The density of states and the spectral shift density of random Schrödinger operators, Rev. Math. Phys. 12 (2000), 807 - 847.

[47] V. Kostrykin and R. Schrader, Global bounds for the Lyapunov exponent and the integrated density of states of random Schrödinger operators in one dimension, J. Phys. A: Math. Gen. 33 (2000), $8231-8240$.

[48] V. Kostrykin and R. Schrader, Regularity of the surface density of states, J. Funct. Anal. 187 (2001), 227 246.

[49] P. Kuchment, Floquet Theory for Partial Differential Equations, Birkhäuser, Basel, 1993.

[50] L.D. Landau and E.M. Lifschitz, Statistical Physics, Pergamon, Oxford, 1980.

[51] D. Lynden-Bell, Statistical mechanics of violent relaxation in stellar systems, Monthly Not. Roy. Astron. Soc. 136 (1967), $101-121$. 
[52] D. Lynden-Bell and R. Wood, The gravo-thermal catastrophe in isothermal spheres and the onset of redgiant structure for stellar systems, Monthly Not. Roy. Astron. Soc. 138 (1968), 495 - 525.

[53] W. Magnus and S. Winkler, Hill's Equation, Interscience, New York, 1966.

[54] Lj. Milanović, H.A. Posch, and W. Thirring, Statistical mechanics and computer simulation of systems with attractive positive powerlaw potentials, University of Vienna preprint, 2001.

[55] G.D. Mahan, Many-Particle Physics, Kluwer Academic/Plenum Pub., New York, 2000.

[56] S. Nakamura, A remark on the Dirichlet-Neumann decoupling and the integrated density of states, J. Funct. Anal. 179 (2001), $136-152$.

[57] J. von Neumann, Thermodynamik quantenmechanischer Grössen, Gött. Nachr. (1927) 273 - 291.

[58] J. von Neumann, Beweis des Ergodensatzes und des H-Theorems in der neuen Mechanik, Z. Phys. 57 (1929), $30-70$.

[59] R. Newton, Bloch-wave scattering by crystal defects, J. Math. Phys. 32 (1991), 551 - 560.

[60] W.B. Nurdin and K.-D. Schotte, Dynamical temperature for spin systems, Phys. Rev. E 61 (2000), 3579 3582.

[61] L. Pastur and A. Figotin, Spectra of Random and Almost-Periodic Operators, Springer, Berlin, 1992.

[62] W. Pauli and M. Fierz, Über das H-Theorem in der Quantenmechanik, Z. Physik 106 (1937), 572 - 587.

[63] T. Padmanabhan, Statistical mechanics of gravitating systems, Phys. Rep. 188 (1990), 285 - 362.

[64] F. Reif, Fundamentals of Statistical and Thermal Physics, McGraw-Hill, New York, 1965.

[65] D. Robert, Asymptotique de la phase de diffusion a haute energie pour des perturbations du Laplacien, Ann. Sci. Ec. Norm. Super., IV. Ser. 25, No.2 (1992), 107 - 134.

[66] D. Ruelle, Statistical Mechanics, Benjamin, New York, 1969.

[67] H. Rugh, Dynamical approach to temperature, Phys. Rev. Lett. 78 (1997), 772 - 774.

[68] H. Rugh, A geometric, dynamical approach to temperature, J. Phys. A: Math. Gen. 31 (1998), $7761-7770$.

[69] M. Schmidt, R. Kusche, W. Kronmüller, B. von Issendorff, and H. Haberland, Experimental determination of the melting point and heat capacity for a free cluster of 139 sodium atoms, Phys. Rev. Lett. 79 (1997), 99 -102 .

[70] M. Schmidt, R. Kusche, B. von Issendorff, and H. Haberland, Irregular variations in the melting point of size-selected atomic clusters, Nature 393 (1998), 238 - 240.

[71] R. Schrader, High energy behavior for non-relativistic scattering by stationary external metrics and YangMills potentials, Z. Phys. C, Particles and Fields, 4 (1980), 27 - 36.

[72] R. Schrader and M.E. Taylor, Small $\hbar$ asymptotics for quantum partition functions associated to particles in external Yang-Mills potentials, Commun. Math. Phys. 92 (1984), 555 - 594.

[73] R. Schrader and M.E. Taylor, Semiclassical asymptotics, gauge fields and quantum chaos, J. Funct. Anal. 83 (1989), 258 - 316.

[74] M.E. Taylor and A. Uribe, Semiclassical spectra of gauge fields, J. Funct. Anal. 110 (1992), 1 - 46.

[75] W. Thirring, Systems with negative specific heat, Z. Phys. 235 (1970), 339 - 352.

[76] A. Wehrl, General principles of entropy, Rev. Mod. Phys. 50 (1978), 221 - 260.

VADIM KOSTRY KIN, FraUnHOFER-INSTITUT FÜR LASERTECHNIK, STEINBACHSTRASSE 15, D-52074 AACHEN, GERMANY

E-mail address: kostrykin@t-online.de, kostrykin@ilt.fhg.de

Robert Schrader, Institut FÜr Theoretische Physik, Freie Universität Berlin, ArniMALLEE 14, D-14195 BERLIN, GERMANY

E-mail address: schrader@physik. fu-berlin.de 